OPEN ACCESS

Edited by:

Olinga Taeed,

Enterprise and Governance,

United Kingdom

Reviewed by:

Alexander Vladimirovich Bogdanov, Saint Petersburg State University,

Russia

Chaitan Baru,

California College San Diego,

United States

*Correspondence:

Jens Ducrée

jens.ducree@dcu.ie

Specialty section:

This article was submitted to

Blockchain for Science,

a section of the journal

Frontiers in Blockchain

Received: 20 November 2020 Accepted: 28 January 2021

Published: 07 May 2021

Citation:

Ducrée J, Etzrodt M, Bartling S, Walshe $R$, Harrington $T$, Wittek $N$, Posth S, Wittek K, lonita A, Prinz W,

Kogias D, Paixão T, Peterfi I and Lawton J (2021) Unchaining Collective Intelligence for Science, Research, and

Technology Development by

Blockchain-Boosted

Community Participation.

Front. Blockchain 4:631648.

doi: 10.3389/fbloc.2021.631648

\section{Unchaining Collective Intelligence for Science, Research, and Technology Development by Blockchain-Boosted Community Participation}

\author{
Jens Ducrée ${ }^{1 *}$, Martin Etzrodt ${ }^{2}$, Sönke Bartling ${ }^{3}$, Ray Walshe ${ }^{4}$, Tomás Harrington ${ }^{5}$, \\ Neslihan Wittek ${ }^{6}$, Sebastian Posth ${ }^{7}$, Kevin Wittek ${ }^{8}$, Andrei lonita ${ }^{9}$, Wolfgang Prinz ${ }^{9,10}$, \\ Dimitrios Kogias ${ }^{11}$, Tiago Paixão ${ }^{12}$, losif Peterfi ${ }^{13}$ and James Lawton ${ }^{13}$
}

${ }^{1}$ School of Physical Sciences, Dublin City University, Dublin, Ireland, ${ }^{2}$ Akasha Foundation, Zug, Switzerland, ${ }^{3}$ Blockchain for Science GmbH, Berlin, Germany, ${ }^{4}$ School of Computing, Dublin City University, Dublin, Ireland, ${ }^{5}$ Innovation, Technology and Operations Management Group, Faculty of Social Sciences, Norwich Business School, University of East Anglia, Norwich, United Kingdom, ${ }^{6}$ Department of Biopsychology, Faculty of Psychology, Ruhr University Bochum, Bochum, Germany, ${ }^{7}$ Posth Werk BV, Leiden, Netherlands, ${ }^{8}$ Institute for Internet Security, Westphalian University of Applied Sciences, Gelsenkirchen, Germany, ${ }^{9}$ Fraunhofer Institute for Applied Information Technology (FIT), Sankt Augustin, Germany, ${ }^{10}$ Faculty of Electrical Engineering and Information Technology, Aachen, Germany, ${ }^{11}$ Department of Electrical and Electronics Engineering, University of West Attica, Egaleo, Greece, ${ }^{12}$ Instituto Gulbenkian de Ciência, Rua da Quinta Grande 6, Oeiras, Portugal, ${ }^{13}$ Max Planck Digital Library (MPDL), Max Planck Society, München, Germany

Since its launch just over a decade ago by the cryptocurrency Bitcoin, the distributed ledger technology (DLT) blockchain has followed a breathtaking trajectory into manifold application spaces. This study aper analyses how key factors underpinning the success of this ground-breaking "Internet of value" technology, such as staking of collateral ("skin in the game"), competitive crowdsourcing, crowdfunding, and prediction markets, can be applied to substantially innovate the legacy organization of science, research, and technology development (RTD). Here, we elaborate a highly integrative, communitybased strategy where a token-based crypto-economy supports finding best possible consensus, trust, and truth by adding unconventional elements known from reputation systems, betting, secondary markets, and social networking. These tokens support the holder's formalized reputation and are used in liquid-democracy style governance and arbitration within projects or community-driven initiatives. This participatory research model serves as a solid basis for comprehensively leveraging collective intelligence by effectively incentivizing contributions from the crowd, such as intellectual property work, validation, assessment, infrastructure, education, assessment, governance, publication, and promotion of projects. On the analogy of its current blockbusters like peer-to-peer structured decentralized finance ("DeFi"), blockchain technology can seminally enhance the efficiency of science and RTD initiatives, even permitting to fully stage operations as a chiefless decentralized autonomous organization (DAOs).

Keywords: token economy, cryptoeconomy, consensus, reputation systems, liquid democracy, participatory research, collective intelligence, wisdom of the crowd 


\section{INTRODUCTION}

From a bigger perspective, science, research, and technology development (RTD) pursues the overarching goal of generating beneficial knowledge that aims to contribute to the good of mankind. Basic science is driven by the quest for learning and understanding as a core trait of human nature; often pursuing commercial objectives, technology improves the quality of our lives, for example, in terms of addressing fundamental needs in food, health, and education, or providing convenience such as water, energy, transportation, automation, communication, and entertainment.

While, at least in the public eye, major breakthroughs are often linked to a single person, practically all historical achievements in science and RTD are deeply rooted in the foundational work of a community which produced the fundamental research as well as enabling methods, equipment, and infrastructure within a supporting culture and, finally, a pool of highly qualified and motivated talent stimulating productivity within a competitive environment.

Generation of basic knowledge is presently, for the most part, sponsored by governmental organizations and foundations to address health, economic, scientific, societal, ethical, and environmental topics and issues, to boost prestige through high-impact publicity, or by businesses to gain a competitive edge for eventually enhancing their profits. Involvement of individuals in such initiatives may be motivated by a variety of objectives, ranging from career opportunity, reputation, social recognition, creativity, altruism, and monetary rewards.

Recent decades have seen a strong trend toward globalization that has been enabled by sophisticated networks of logistics and highly intertwined global supply chains which are meticulously coordinated by transnationally operating information and communication technology (ICT). With the wide-scale penetration of the Internet, ensued by the availability of cloud computing and artificial intelligence (AI), possibly accessed by science as a service model (Yang, 2020), and 5G networks for real-time applications, even in remote and economically disadvantaged locations, access to and creation of knowledge has already reached historically unprecedented levels.

The recent past has also seen game-changing advances in ubiquitous/additive manufacturing (UM/AM), primarily by threedimensional (3D) printing (Chen, 2020) or open-source platforms, for example, in software, ${ }^{1}$ electronics, ${ }^{2}$ and microsystems (Ducrée et al., 2020b); virtualization, for instance, through "digital twins" (Grieves and Vickers, 2017), open-access facilities for prototyping,

${ }^{1}$ Linux.org. Available at: https://www.linux.org/ (Retrieved August 13, 2020). ${ }^{2}$ Arduino is an Open-Source Electronics Platform Based on Easy-To-Use Hardware and Software Available at: https://www.arduino.cc/ (Retrieved August $11,2020)$ characterization, and application development; ${ }^{3-7}$ and data on demand. $^{8}$ The wide-scale availability of these novel resources will progressively empower individuals from the crowd, such as the "citizen scientist" ${ }^{\text {"9,10; }}$; or "garage entrepreneur," to create, customize, or sell many digital and physical goods from and to practically anywhere. In addition, the emergence of redistributed manufacture (RDM) echoes that production is becoming increasingly decentralized, for example, even in the highly regulated pharmaceutical industry ("Portable, continuous, miniature and modular - the pharma factory of the future," Congdon, 2020) ${ }^{11}$ and aerospace (Birch, 2019).

Open science and community-based participatory research (CBPR) for socio-environmental issues is another area of focus (Kondo et al., 2019). Here, discovering and sharing goals that actors with different interests could tackle together (a "transcend" method), in addition to ethical equity, fair access to data, and dialogue, represent essential components. The constructive interplay of these strong trends sets the path for a democratization of an inclusive science and RTD landscape where a wide range of actors, whether institutions or freelancers, ${ }^{12-14}$ can decisively contribute and receive their fair share in value creation.

CBPR has been regarded as the gold standard for equitable and partnered research in traditional communities (Winterbaueret al., 2016). In capturing the collaborative process between community-based organizations and academic investigators, CBPR models have demonstrated the potential to make research more responsive to existing needs, and to improve a community's ability to address a range of common issues Weiner and McDonald (2013). Such CBPR approaches can be enhanced by token systems ("tokenization") which, on one hand, cryptoeconomically incentivize efficient crowdsourcing of collective intelligence (Malone and Bernstein, 2015), ${ }^{15}$ while, on the

${ }^{3}$ Biolabs-Build Your Biotech. Available at: https://www.biolabs.io/ (Retrieved September 28, 2020).

${ }^{4}$ Fab Labs. Available at: https://www.fablabs.io/ (Retrieved July 24, 2020).

${ }^{5}$ ECL-Emerald Cloud Labs. Available at: https://www.emeraldcloudlab.com/ (Retrieved September 28, 2020).

${ }^{6}$ UniteLabs-Digital Doesn't Have to be Complicated. Available at: https:// unitelabs.ch/ (Retrieved September 28, 2020).

${ }^{7}$ BosLab-Our Mission is to Democratize Biotechnology in Our Community (Retrieved January 04, 2021).

${ }^{8}$ ARCTORIS-Drug Discovery Data on Demand. Available at: https://www. arctoris.com/ (Retrieved September 28, 2020).

${ }^{9}$ DITOS Consortium and We Observe Consortium (2019). Making Citizen Science Work: Innovation Management for Citizen Science. DITOS Policy Brief 6. Available at: https://discovery.ucl.ac.uk/id/eprint/10073927/1/Albert_Innovationmanagement.pdf (Retrieved January 4, 2021).

${ }^{10}$ Foldit is a Revolutionary Crowdsourcing Computer Game Enabling You to Contribute to Important Scientific Research. (Retrieved July 24, 2020).

${ }^{11}$ Portable, Continuous, Miniature and Modular-The Pharma Factory of the Future. Manufacturing Chemist. Available at: https://www.manufacturingchemist. com/news/article_page/Portable_continuous_miniature_and_modular_the_ pharma_factory_of_the_future/117046.

${ }^{12}$ OrbiNetwork-The Future of Work. Available at: https://orbi.network/ (Retrieved September 10, 2020).

${ }^{13}$ Gitcoin-Crowdfunding and Freelance Developers for Open Source Software Projects (Retrieved July 24, 2020).

${ }^{14}$ AnyTask. Available at: https://anytask.com/ (Retrieved October 4, 2020).

${ }^{15}$ Collective Intelligence. Available at: https://en.wikipedia.org/wiki/Collective_intelligence. 


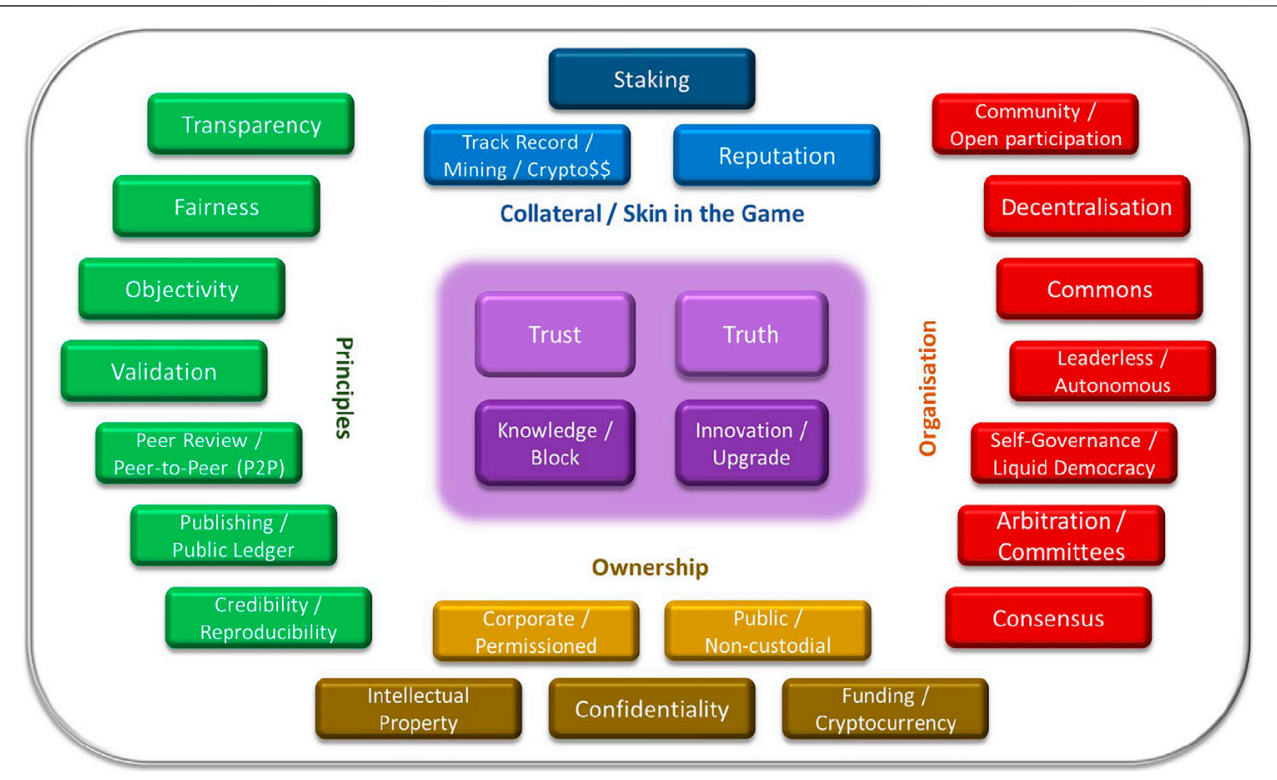

FIGURE 1 | Buzz words in science and RTD which directly connect to equivalent elements in blockchain technology. The central objective is to create blocks of trust and truthful knowledge/innovation and log them a public or permissioned ledger. Credibility of contributions is rewarded and staked by work efforts and reputation. Ownership and confidentiality of results depend on the source of funding, and may be kept as corporate secret or made public. The construct is framed by mechanisms to assure quality and credibility of results on the left and involvement of the community on the right-hand side.

other hand, request cryptoassets as collateral ("skin in the game") to assure good quality of contributions.

This way, such blockchain-based crypto-economical tools, possibly in tandem with the aforementioned CBPR, can also be key to address the notorious reproducibility crisis of science (Baker, 2016; Voelkl et al., 2020). The pervasive failure to replicate previous findings often roots in inappropriate practices of science, for example, poor experimental design leading, almost unavoidably, to variability between the groups (Resnik and Shamoo, 2017), hypothesizing after the results are known ("HARKing”) (Kerr, 1998; Rubin 2017), selective analysis and reporting (Dwan et al., 2008; Page et al., 2014; Open Science, 2015), $p$ value hacking (Smith and Ebrahim, 2002; Head et al., 2015), and missing raw data (Zuiderwijk and Spiers, 2019; Miyakawa, 2020). Freedman et al. (2015) demonstrated that in the United States, the high rate of irreproducibility, estimated at $50 \%$ in preclinical research, induced high economical loss of approximately USD28 billion per annum. In addition to waste of money and time, the poor reproducibility of data tends to markedly demotivate the idealistic scientific community.

Furthermore, the recent shift toward open access in scientific publishing, even though widely regarded as worthwhile and necessary, has led to detrimental side effects; driven by profit, a slew of open-access journals publish quasi any submission because the costs have entirely shifted from the readers or subscribing libraries to the authors. This unintentional, very counterproductive development has led to a noticeable rise of such rather predatory journals with substandard editorial boards, peer reviewers, and papers, and even to the loss of parts of the scientific record when their entirely profit-focused publishing houses suddenly dissolve. This severe problem could well be addressed by increased transparency of the peer-review system, as well as a robust identity and reputation systems of authors, reviewers, and journals, all of which can be successfully addressed by blockchain technologies, as described below.

This study proposes a radically novel decentralized concept utilizing blockchain mechanisms based on reputation systems, betting, secondary markets, and social networking to deliver well-qualified consensus for (best possible) "truth" (as validated according to commonly accepted scientific procedures) on delivery and assessment of plans, work, and forecasts, to eventually amend "blocks" of scientifically or commercially valuable artifacts, knowledge, or know-how to the public or corporate ledger of science and RTD, respectively. Figure 1 lists critical modules of science and RTD that can be mapped to blockchain technology to seminally update the way science and RTD have been organized over the last centuries, to ultimately confer optimum benefit to people, societies, and economies.

\section{BLOCKCHAIN}

Beyond its foundation in computer science, cryptography, and finance, blockchain technology is not monolithic; its sophistication and application development now involve concepts from a wide range of disciplines encompassing 


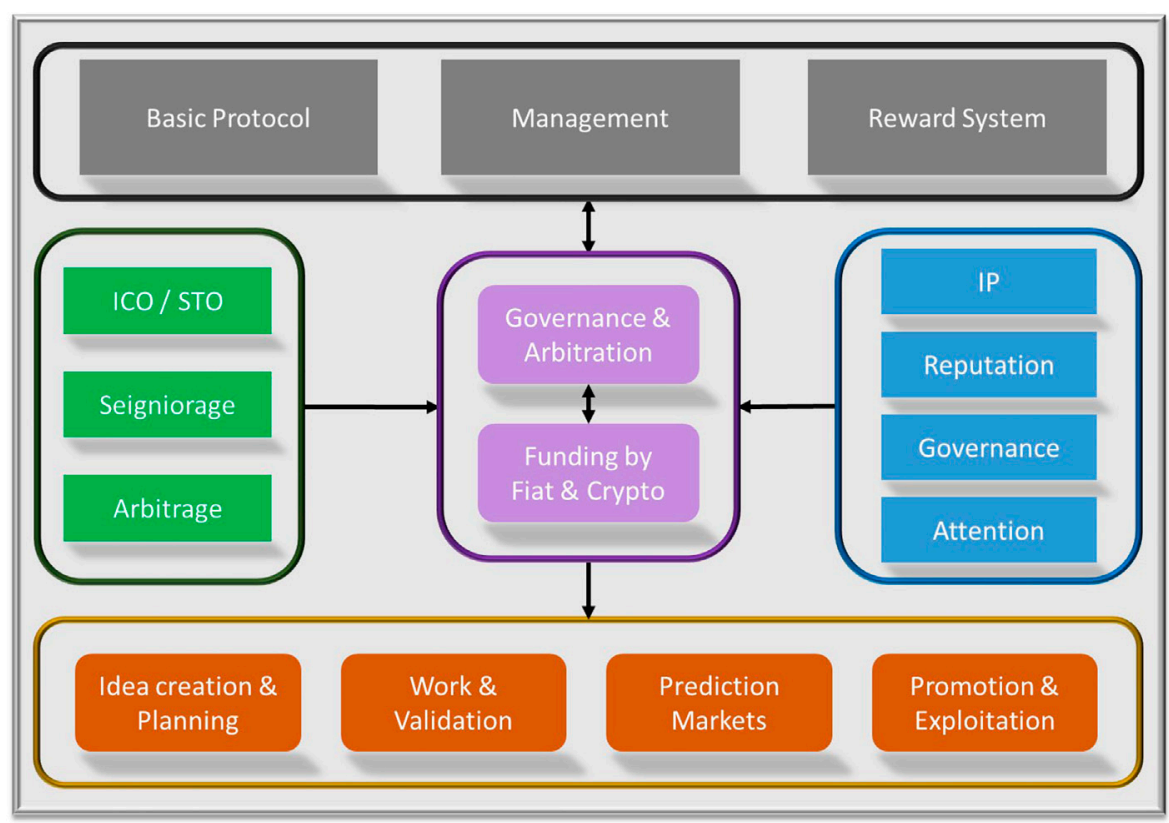

FIGURE 2 | Organization of science and RTD by a token-based crypto-economy. A white paper documents a basic protocol, management structures, and reward systems which are encoded and executed on a blockchain. Govemance and arbitration following liquid democracy schemes are at the heart of decision flexible updating of rule sets, directing, and conflict resolution. Blockchain mechanisms such as ICOs/TOS/STOs, seigniorage and arbitrage generate conventional, fiat-convertible funding. A cohort of crypto tokens can be awarded to users in the crowd, for example, for contributions such as ideas, planning, work, validation, forecasting, exploitation, and even to externals by learning about or referencing the project, and thus raise attention. The recipients might deploy these rewards to increase voting rights in the liquid democracy govemance, to polish up their curriculum vitae (CV), to claim ownership in intellectual property $(\mathrm{IP})$, and, in a potential secondary market, trade them into other crypto assets.

economics, game theory, banking, risk management, data science, education, law, administration, political science, psychology, ethics, arts, and social sciences. While gaining a thorough understanding of blockchain tends to be somewhat challenging, its diverse contributions bear strong potential to produce disruptive ideas, concepts, and solutions that continuously drive the evolution of the overarching technology. In the following sections, we provide an insight into the different facets of this distributed ledger technology (DLT) (Hodgson, 2016) to then lay the ground for substantially improving legacy organization and processes in science and RTD.

\section{Digital Currency}

Blockchain originates from a white paper, authored by the pseudonymous person or group "Satoshi Nakamoto," which launched the virtual currency "Bitcoin" in 2008/2009 (Nakamoto, 2009; Swan, 2015; Kenton, 2020). ${ }^{16}$ By employing a computational "proof of work" (PoW) in conjunction with incentivization for honest participation, this document described the world's first realization of a peer-to-peer version of electronic cash that offers algorithm-enforced scarcity, solves the double-spending problem of digital assets, and allows online payments to be sent directly from one party to another without going through a financial institution.

Integrity and consensus on amending new "blocks" is achieved through a clever combination of cryptography and a decentralized

${ }^{16}$ Bitcoin Is an Innovative Payment Network and a New Kind of Money. Available at: https://bitcoin.org/ (Retrieved August 14, 2020). peer-to-peer network of miners to validate and nodes to store a common, tamper-free, immutable, and time-stamped distributed ledger (file). The protocol runs without intermediaries, for example, a central bank, whose monetary policies may be directed by short-term political objectives of the ruling administration, rather than protecting the interest of people, especially depositors, against inflation ${ }^{17}$ and devaluation; uniquely, no physical goods such as bills, coins, or precious metals are issued, stored, split, or transported, thus drastically reducing cost of ownership, security, and usage.

Bitcoin and many other cryptocurrencies may be traded on conventional or decentralized exchanges, ${ }^{18}$ "hodled," 19 that is, held on the long term in the prospect of large future profit or increased utility, saved to protect wealth against inflation or for long-term gains in on- or offchain wallets or vaults, or used to buy real-world goods and to access services with an expanding number of merchants. ${ }^{20}$ By the time of writing, Bitcoin has reached a total market value far in the region of 1,000 billion USD, thus being ranked the world's sixth largest currency (depending on the referenced category of money supply). It is followed

\footnotetext{
${ }^{17}$ This philosophy is also carved into Bitcoin's genesis block stating "The Times $03 /$ Jan/2009 Chancellor on brink of second bailout for banks." issued in the middle of the 2008-2009 financial crisis.

${ }^{18}$ Decentralized Exchange (DEX). Available at: https://en.wikipedia.org/wiki/ Decentralized_exchange (Retrieved August 7, 2020).

${ }^{19}$ Hodl. Available at: https://en.wikipedia.org/wiki/Hodl (Retrieved August 18, 2020).

${ }^{20}$ What to Buy with Bitcoins. Available at: https://cointelegraph.com/bitcoin-forbeginners/what-can-i-buy-with-bitcoins (Retrieved August 18, 2020).
} 
by Ethereum (ETH) (O'Neal 2020), ${ }^{21}$ the global payment network Ripple (XRP) ${ }^{22}$ with about 45 and 13.2 billion USD, respectively, and a host of further, often special purpose, "altcoins."

\section{Computing Power}

Trust in Bitcoin is established through demanding PoW, that is, staking huge computational power in a race to unravel a cryptographic "puzzle" (or, more accurately, by finding a partial hash collision through brute force), which nowadays requires designated, application-specific integrated circuits (ASICs) that frequently concentrate at larger scale mining "farms." Blockchains are commonly designed to be "Byzantine fault tolerant" (BFT), ${ }^{23}$ that is, only an entity able to control a significant fraction (arguably specified between $1 / 3$ and 51\% under real-world circumstances) of the so-called hash rate (or alternative resource) would have a realistic chance to manipulate and thus devastatingly compromise trust in the blockchain; such attacks are efficiently counteracted by attractive rewards for mining or staking. It is widely accepted that only seminal enhancements of hash rates, for example, by next-generation quantum computing, might pose a serious challenge to securing blockchains (more precisely: public PoW-based chains that work on hash-collision-based cost functions) in the longer term future.

\section{Programmable Money}

Another milestone flanking the emergence of blockchain technology was the introduction of "smart contracts" which allowed the development of decentralized apps ("DApps") that are equipped with a locally running user interface (UI), with the business logic executed in a decentralized fashion, for example, on the Ethereum Virtual Machine (EVM) as part of a network (Buterin, 2014). In this "Web 3.0"24 technology launched in 2014, the execution of transactions on assets can be coupled to conditions represented by code that is permanently engraved and verified on the blockchain. Such programmability of a trusted digital currency has given DLT another tremendous, at times somewhat overhyped boost in the second half of the 2010s, in particular in the field of decentralized finance (“DeFi") (Austin, 2020), which is presently spurred by hot topics like "yield farming"/"liquidity mining.",25-31

\footnotetext{
${ }^{21}$ Ethereum is a Global, Open-Source Platform for Decentralized Applications. Available at: https://ethereum.org/ (Retrieved August 14, 2020).

${ }^{22}$ Ripple. Available at: https://ripple.com/ (Retrieved August 14, 2020).

${ }^{23}$ Byzantine Fault. Available at: https://en.wikipedia.org/wiki/Byzantine_fault (Retrieved September 14, 2020).

${ }^{24}$ Note that there are various conflicting definitions exist for the terms "Web3" or "Web 3.0."

${ }^{25}$ What is Yield Farming? The Rocket Fuel of DeFi. Available at: https://www. coindesk.com/defi-yield-farming-comp-token-explained (Retrieved August 17, 2020).

${ }^{26}$ Compound. Available at: https://compound.finance/ (Retrieved August 17, 2020).

${ }^{27}$ Uniswap-Automated Liquidity Protocol. Available at: https://uniswap.org/ (Retrieved August 17, 2020).

${ }^{28} \mathrm{Balancer}$ is a Protocol for Programmable Liquidity. Available at: https://balancer. finance/ (Retrieved August 17, 2020).

${ }^{29}$ Synthetix - A Protocol for Trading Synthetic Assets on Ethereum. Available at: https://www.synthetix.io/ (Retrieved August 17, 2020).

${ }^{30}$ Aave-The Money Market Protocol. Available at: https://aave.com/ (Retrieved September 7, 2020).

${ }^{31}$ SushiSwap-An Evolution of Uniswap with SUSHI Tokenomics. Available at: https://sushiswap.org/ (Retrieved September 7, 2020).
}

High volatility of exchange rates with fiat currencies has been addressed by stablecoins, often soft- or hard-pegged against the US dollar $^{32-34}$. Some companies insure against adverse events ${ }^{35,36}$ and professionally audit smart-contract constructs. ${ }^{37}$ However, enforcement of severe regulation, for example, around know-your-customer ${ }^{38}$ and anti-money-laundering (“AML") compliance policies (KYC) required in conventional financial services, is still regarded as major roadblocks for further land-slide proliferation; while there is significant support for introduction of "crypto" from the corporate sector and important interest groups, increasing support is signaled from government agencies who would need to legally sanction certain steps and support integration with their work flows (Hubbard, 2020; Jones, 2020; Post, 2020). ${ }^{39-41}$ Alternative public blockchain-enabled cryptoassets like Bitcoin, numerous countries are seriously contemplating the introduction of Central Bank Digital Currencies (CBDCs).

\section{Oracles}

Smart contracts may be deemed as blockchain-based algorithms that deterministically trigger transactions, for instance, of cryptocurrencies, upon meeting well-defined conditionals on external input data, referred to as "oracles." Blockchains themselves provide trust on information that can be sourced from its very own digital ecosystem, for example, that a certain date has passed, or a certain amount of funds is available on a given crypto account. However, any real-world data feeds, for example, on stock values, exchange rates, opinion polls, or data obtained from the Internet of Things (IoT), to on-chain decision-making algorithms constitute potential chinks caused by poor quality or even forgery and would thus devastatingly undermine trust in blockchain-controlled processes. Access to reliable off-chain facts by properly vetted and incentivized "reporters" is thus instrumental for the implementation and acceptance of oracle-based smart contracts controlling transactions of value.

There are different types of oracles: ${ }^{42}$ software and hardware oracles receive data from online sources, and real-world information from physical sensors, respectively. In inbound and outbound oracles, digital information is fed

\footnotetext{
${ }^{32}$ MakerDAO. The Maker Protocol: MakerDAO's Multi-Collateral Dai (MCD) System. Available at: https://makerdao.com/en/whitepaper (Retrieved July 24, 2020)

${ }^{33}$ Tether. Available at: https://tether.to/ (Retrieved July 24, 2020).

${ }^{34}$ USD Coin. Available at: https://www.coinbase.com/usdc (Retrieved August 17, 2020).

${ }^{35}$ Opyn-Securing Decentralized Finance. Available at: https://opyn.co/ (Retrieved August 17, 2020).

${ }^{36}$ Nexus Mutual-A People-Powered Alternative to Insurance. Available at: https:// nexusmutual.io/ (Retrieved August 17, 2020).

${ }^{37}$ Quantstamp-Smart Contract Security. Available at: https:/quantstamp.com/ (Retrieved August 17, 2020).

${ }^{38}$ Know Your Customer (KYC). Available at: https://en.wikipedia.org/wiki/Know_ your_customer (Retrieved September 7, 2020).

${ }^{39}$ Government Blockchain Association (GBA). Available at: https://www.gbaglobal. org/ (Retrieved July 24, 2020).

${ }^{40}$ Bundesblock. Available at: https://bundesblock.de/ (Retrieved July 24, 2020).

${ }^{41} \mathrm{EU}$ Blockchain Observatory \& Forum. Available at: https://www. eublockchainforum.eu/ (Retrieved October 9, 2020).

${ }^{42}$ Blockchain Oracles. Available at: https://blockchainhub.net/blockchain-oracles/ (Retrieved July 24, 2020).
} 
to or issued from the blockchain. Consensus-based oracles rely on verification and approval by a cohort of individuals or groups. A range of initiatives already provide various forms of such blockchainconnected oracles (Zhang et al., 2016; Kapilkov, 2020). ${ }^{43-48}$ Especially consensus-based oracles play an important role in this study, as they best reflect the well-proven trust-finding process already underpinning science and RTD.

\section{Prediction Markets}

Uncertainty on future events and developments remains a fundamental issue in our lives, economies, and societies. Examples are weather, climate, natural or manmade disasters, conflicts, health, election results, consumer behavior, financial markets, and economic growth from a macroscopic and corporate angle as well as advancements in science and technology. Undoubtedly, there is a huge value in having prior knowledge about upcoming events, for example, to advise allocation of resources or to preemptively mitigate their expected fallout. Organizations like insurers, investors, governments, and companies rely on thorough data collection and analysis to have the best possible appraisal.

While small panels of pundits may be consulted for their insights, the collective wisdom of the crowds (Surowiecki, 2004; Becker, 2010; Hill and Ready-Campbell, 2011) has often delivered an astonishingly reliable source for predicting the future events, for example, the outbreak of pandemics (McKenna, 2020; van der Waal et al., 2020), possibly because a broad population provides a multitude of information, perspectives, and experiences to average out individual bias. However, it is also reasonable to put higher weight on forecasts uttered by specialists displaying a thoroughly verified "reputation," that is, a proven track record, untarnished credibility, and relevant expertize in the area under consideration for optimizing the accuracy. For example, artificial intelligence (AI)-supported DeFi platforms have been launched, involving reputation-staked collective intelligence of investors from the crowd to direct an open hedge fund. ${ }^{49}$

Its combination of smart contract and trust without intermediaries has been a fertile ground to install several prediction markets on blockchains (Davidov, 2019; Foxley, 2021) ${ }^{50-53}$; these next-

\footnotetext{
${ }^{43}$ The Confidential Consortium Framework (CCF). Available at: https://github. com/microsoft/CCF (Retrieved January 4, 2020).

${ }^{44}$ Oracles. Available at: https://docs.corda.net/docs/corda-os/4.5/key-conceptsoracles.html (Retrieved August 10, 2020).

${ }^{45}$ The Provable ${ }^{\mathrm{TM}}$ Blockchain Oracle for Modern DApps. Available at: https:// provable.xyz/ (Retrieved August 10, 2020).

${ }^{46}$ Aeternity-A Blockchain for Scalable, Secure, and Decentralized æpps. Available at: https://aeternity.com/ (Retrieved August 10, 2020).

${ }^{47}$ Band Protocol-Secure, Scalable Blockchain-Agnostic Decentralized Oracle. Available at: https://bandprotocol.com/. (Retrieved August 17, 2020).

${ }^{48}$ CHAINLINK-Your Smart Contracts Connected to Real World Data, Events and Payments. Available at: https://chain.link/ (Retrieved July 27, 2020).

${ }^{49}$ Numerai-The Hardest Data Science Tournament on the Planet. Available at: https://numer.ai/ (Retrieved August 28, 2020).

${ }^{50}$ Augur -Put Your Skills to the Test and WIN! (Retrieved 24/07/2020).

${ }^{51}$ Stox.com-Predict on the Hottest Markets, Create Your Own Predictions, and Make Profit on One Platform. Available at: https://www.stox.com/ (Retrieved July 27, 2020).

${ }^{52}$ Gnosis.io-Redistribute the Future. Available at: https://gnosis.io/ (Retrieved July 27, 2020).

${ }^{53}$ Sight.pm-Talk Values. Sight Knowledge. Available at: https://sight.pm/ (Retrieved July 27, 2020).
}

generation platforms allow "anyone", including automated market makers (AMMs), to create trade pairs and settle their "bets" at different stages prior to the completion of the event. As any crypto exchange, attracting sufficient liquidity is paramount for the viability of these platforms.

Evidently, precise definition of possible outcomes constitutes a critical prerequisite for setting up and settling the market. There are outcomes that are of binary, that is, yes-no nature, or that can be expressed in exact numbers. Yet, even those events may fall out of a set list of outcomes, for example, by cancellation or changing of goal posts due to unforeseeable force majeure. This may be handled by declaring the bet invalid followed by returning deposits to all stakeholders.

However, even such a decision may be disputable, possibly favoring certain stakeholders while leaving some betters feeling betrayed. In this case, trust in the platform is enhanced by providing a predefined and fair arbitration process. ${ }^{54-56}$ Such consensus finding by broadly accepted dispute resolution schemes will also be required for judging on more complex outcomes, for example, if a "soft" scientific or business goal cannot be expressed in a simple quantitative measure.

The main vulnerability of these blockchain-based betting portals resides in the accountability of oracles reporting on real-world data, that is, they may accidently, erroneously, or deliberately feed inaccurate or untimely information which prompts irreversible "pay outs" linked to unstoppable smart contracts. Blockchain-based prediction markets consequently need to put particular emphasis on trustful input sources; for assuring credibility or "best possible trust," staking "skin in the game" as collateral thus needs to be demanded from reporters, for example, by appropriate (crypto-)assets or formalized reputation, while penalizing deviation from the final consensus. Mechanisms for establishing trustful oracles have been developed, for example, in the context of IoT. ${ }^{57}$

\section{Curation Markets}

Also in response to adverse crosswinds by regulatory agencies challenging the legitimacy of initial coin offerings (ICOs), socalled token bonding curves (TBCs) (Goro, 2018) have been devised to implement legally less-controversial idea markets. ${ }^{58}$ Tokens may be purchased and traded for proposals and ongoing projects; staked crypto deposits are safely stored on the blockchain to guarantee continuous liquidity. Their value is defined by a publicly accessible formula, for instance, with a price proportional to the number of tokens issued at a given point in time; in this exemplary model, investors in these curation markets (de la Rouviere, 2017) thus profit when more tokens circulate at the point of selling than at buying. Like conventional

\footnotetext{
${ }^{54}$ Organizations of the Future Run on Aragon. Available at: https://aragon.org/ (Retrieved July 24, 2020).

${ }^{55}$ Telos Blockchain Network Arbitration Rules and Procedures. Available at: https://resources.telosfoundation.io/governance_documents/TBNARP_Adopted_ 2018-10-26.pdf (Retrieved July 24, 2020).

${ }^{56}$ Kleros. Available at: https://kleros.io/ (Retrieved January 4, 2020).

${ }^{57}$ IOTA Foundation-Redifining Trust, Value and Ownership. Available at: https:// www.iota.org/ (Retrieved September 27, 2020).

${ }^{58}$ Ideamarket.io. Available at: https://ideamarket.io/ (Retrieved July 24, 2020).
} 
betting, stockbrokers or currency exchanges, a fraction of the revenues may be commissioned in a well-defined fashion to fund the project activities and to support the creation of trustful prediction markets that can wisely inform decision-making.

To avoid Ponzi schemes, rules must be established for an end game when the project has reached its milestones, for example, delivered commercially for the conversion of virtual token investments into real-world value. As direct pecuniary remuneration may raise the ire of federal authorities who might categorize them as securities to which rather tight regulation would apply to, tokens might be converted into formalized reputation or voting rights in community-driven initiatives seeking related expertise.

\section{Incentivization of Contributors}

The success of community-based approaches involving collective intelligence is intimately hinged on engagement of a critical mass of participants. The same applies to blockchain technologies themselves, which can only thrive if a substantial number of active users and providers of independent nodes perform mining, maintenance, and upgrades to sustain the network.

A variety of schemes have been applied to stimulate employee participation in traditional companies; interestingly, it turns out that accompanying recognition and acknowledgment of quality work has a stronger long-term effect on employee motivation and performance than (stand-alone) bonuses and pay rises (Daly, 2020). Incentivization or collaboration by blockchain-secured token systems has already been implemented by several big corporations. For instance, the Enterprise Ethereum Alliance (EEA) (Cant, 2019), backed by multinationals like Microsoft and Intel, issues reward and reputation tokens to stimulate collaboration on activities like editing and contributing to specifications, and adding code in software development. Penalty tokens may reflect lack of engagement or infringement of project delivery within target budgets, timelines, and specifications. Spotting bugs and other flaws might be incentivized by posting bounties, ${ }^{59,60}$ which may also be open to the public and may be rewarded through centralized or decentralized decision-making mechanisms.

\section{Tokenization of Assets}

The ownership of certain unique assets such as real estate, vehicles, memorabilia, antiques, artwork (Blenkinsop 2020) 61,62 $^{6}$ and other intellectual property (IP) representing intangible creations of human creativity $^{63}$ is normally handled by publicly sanctioned administrative entities, for example, land

\footnotetext{
${ }^{59}$ Bounty0x-Earning Crypto Made Simple. Available at: https://bounty0x.io/ (Retrieved July 27, 2020).

${ }^{60}$ The Bounties Network - Changing the Way Communities Collaborate. Available at: https://www.bounties.network/ (Retrieved December 2, 2020).

${ }^{61} 4$ ART Technologies. Available at: https://www.4art-technologies.com/ (Retrieved November 10, 2020).

${ }^{62}$ Aetsoft-Blockchain Development for Real Estate. Available at: https://aetsoft. net/solutions/blockchain-real-estate/ (Retrieved November 11, 2020).

${ }^{63}$ Intellectual Property IP. Available at: https://en.wikipedia.org/wiki/Intellectual_ property (Retrieved 13/08/2020).
}

registries or patent and trademark offices. Traditionally, the final proof of ownership is still linked to the possession or filing of a paper document that needs to be managed, transferred, archived, and kept accessible in a manner that is highly resilient to forgery, theft, disasters, and physical decay over long, even historic periods of time.

As opposed to money or commodities like precious metals or natural resources, this specific class of goods is not directly interchangeable, that is, title of such assets may need to be swapped, for example, through cumbersome escrow and distribution mechanisms. For instance, the deeds of a house might not be divided in as straight-forwardly as 100 units of a fiat currency might be split into several bills or coins that can be passed out to individuals. Also, a trustful, uninterrupted record of previous ownership, ideally tracing back to the origin, minimizes chances of legal disputes and counterfeit, and thus constitutes a high value.

The enormous potential of the distributed, unstoppable, timestamped, cryptographically secured and thus inviolable public ledger to document non-repudiable ownership of this type of noninterchangeable assets in an "Internet of value" is very evident. The so-called non-fungible tokens (NFTs, known as ERC-721 tokens (Entriken et al., 2020) on the Ethereum blockchain) have been implemented on blockchains (Laurent et al., 2018). ${ }^{64}$ These NFTs have first been utilized for trading unique digital artwork as part of online gaming; ${ }^{65}$ by now, several blockchain initiatives have extended the concept of NFTs to realworld assets (Martin, 2020; Peng 2020a) ${ }^{62,66,67}$ through digital security tokens.

Other projects also target recording and providing selective access to well-verified professional and educational qualifications on designated blockchains (Roberts, 2019), for example, to facilitate admission to academic programs, for streamlining corporate recruitment and for optimizing human resources (HR). ${ }^{68}$

\section{Funding}

In addition to conventional sponsorship by investors, foundations, public agencies, and charities through injection of fiat currency, blockchain technologies offer bespoke funding mechanisms which are linked to the trust without middlemen conferred by the decentralized, cryptosecured ledger and its smart contracts. Funding can, for instance, be raised by minting project-specific digital "coins," for example, so-called ERC-20 tokens ${ }^{69}$ on Ethereum (Buterin, 2014), which may be (pre-)mined and traded according to a transparent protocol. The value of these tokens is then freely determined by market dynamics. On top

\footnotetext{
${ }^{64}$ NonFungible. Available at: https://nonfungible.com/ (Retrieved 10/10/2020). ${ }^{65}$ Cryptokitties. Available at: https://www.cryptokitties.co/ (Retrieved 24/07/2020). ${ }^{66}$ SharpShark. Available at: https://sharpshark.io/ (Retrieved 09/08/2020).

${ }^{67}$ RealT-Fractional and Frictionless Real Estate Investing. Available at: https:// realt.co/ (Retrieved September 7, 2020).

${ }^{68}$ Experis Ireland. How Blockchain Can Disrupt Recruitment and HR. Available at: https://www.experis.ie/blog/2019/06/how-blockchain-can-disrupt-recruitmentand-hr (Retrieved 14/08/2020).

${ }^{69}$ ERC-20 Tokens, Explained. Available at: https://cointelegraph.com/explained/ erc-20-tokens-explained (Retrieved 04/01/2021).
} 
of their financial value, these crypto-currencies might provide a "proof of stake" (PoS), for example, for voting on upgrades or seats on arbitration panels, or for settling utility (transaction) fees charged to sustain the ecosystem of the underlying blockchain.

Trust in such monetary seigniorage, ${ }^{70}$ that is, the difference between the value of new money and the cost of their creation, is best supported best by open-source release of code, proper documentation, and credible validation of progress to avoid scams. Also "gaming" of coins and tokens by speculators poses a mission-critical risk to trust in projects, which is particularly pronounced with tiny trade volumes and limited numbers of independent stakeholders in the market.

Financial authorities have frequently categorized cryptoassets issued through initial coin offerings (ICOs) for funding blockchain projects as equivalent to securities, which would require compliance to stringent rules similar to initial public offerings (IPOs) of "shares" on traditional (centralized) stock exchanges. Such classification requires enormous efforts on compliance. Alternative models have been developed for tokens not representing classical assets and ownership. They may be offered in initial (security) token offerings, ITOs or STOs, respectively, and may then be traded in secondary markets. Also, the issue of legally sound incorporation of businesses ("LAOs") using cryptoeconomic mechanisms has been elaborated. ${ }^{71}$ The attraction of such democratized decision-making organization to venture capital (VC) funding remains to be seen.

\section{Organization}

As it has already surfaced in the previous sections, the availability of smart contracts on blockchains opens manifold avenues for rethinking classical approaches to the organization of projects. From a certain point of view, novel implementations seek to extend trust finding by decentralization, majority vote, and PoW/PoS from the core of blockchain to the entire management structure. This applies to commercial projects, for example, to direct management and investment decisions in DeFi by sourcing the wisdom of the crowd; such decentralized governance, for example, as implemented by liquid (delegated) democracy ${ }^{72}$ -based establishment of consensus, also invigorates trust and community spirit in initiatives pursuing "Commons," that is, shared goals on advancement of blockchain technologies or for the good of mankind.

Voting rights of stakeholders might be weighted by the amount (crypto-)assets, which in addition to purchase, may be earned by quality contributions to the entire gamut of the project such as work, validation, assessment, management, promotion,

\footnotetext{
${ }^{70}$ Seigniorage. Available at: https://en.wikipedia.org/wiki/Seigniorage

${ }^{71}$ OpenLaw. Available at: https://www.openlaw.io/ (Retrieved 17/08/2020).

${ }^{72}$ Liquid Democracy. Available at: https://en.wikipedia.org/wiki/Liquid_ democracy (Retrieved 12/08/2020).
}

community building, forecasts, arbitration, governance, and by running infrastructure and further development of the underlying blockchain (Moloch, MetaCartel, and Cardano).

As the pinnacle of such developments, so-called decentralized autonomous organizations (Siegel 2016; Vigna 2016; MakerDao, 2020 $)^{73-77}$ have been launched in the second half of the 2010s; these "DAOs" encode the complete organizational structure of a cooperative project into (a set of) smart contracts. DAOs are rapidly evolving into social tools and organizations that pursue a collective agenda for the benefit of the community or societies in general (Ducrée et al., 2020). ${ }^{78}$ While some of these community-run, smartcontract constructs have exposed major weaknesses or failed (Siegel, 2016; Dale and Foxley, 2020; Foxley, 2020), for example, due to vicious cyber-attacks or exploitation enabled by flawed or ill-coded smart contracts, several DAOs, some of them attracting up to billion-dollar volumes of crowdfunding (Peng 2020b), ${ }^{13,79}$ have been successfully established in the meantime. The launch of DAOs and DApps is frequently accompanied by posting bounties for spotting bugs and vulnerabilities in the code.

\section{Resources Sharing and Circular Economy}

Depending on the field the blockchain technology is applied to, organizations can define a specific set of smart-contractimplemented governance rules that suit the objectives of the supporting communities. These guidelines may improve, for instance, fairness, create equal opportunity, or minimize environmental footprint, for example, by blockchain-enabled sharing or even circular economies.

Examples for governing shared resources by DAOs are computing storage and processing, vehicles, office space, laboratories, and virtual resources. Blockchain-leveraged tokenization may incentivize actions that bring value to communities, thereby shifting the focus on reutilizing idle or available resources which otherwise are very difficult to account for and are usually ignored.

\section{Current Advances}

In addition to coordination with governments and public agencies, which is supported by a rising number of large-scale corporate

\footnotetext{
${ }^{73}$ Decentralized Autonomous Organization. Available at: https://en.wikipedia.org/ wiki/Decentralized_autonomous_organization (Retrieved 14/08/2020).

${ }^{74}$ Moloch DAO Explained: Using Self-Interest to Ethereum's Advantage. Available at: https://concourseopen.com/blog/moloch-dao-explained/ (Retrieved 18/ 08/2020).

${ }^{75}$ DAOstack Is an Open Source Project Advancing the Technology and Adoption of Decentralized Governance. Available at: https://daostack.io/ (Retrieved 17/ 08/2020).

${ }^{76}$ The Genesis DAO. Available at: https://daostack.io/genesis (Retrieved 18/08/ 2020).

${ }^{77}$ DAOfest. Available at: https://www.daofest.io/ (Retrieved 17/08/2020).

${ }^{78}$ MetaCartel Is a Ecosystem of Creators and Operators Building Decentralized Applications (DApps). Available at: https://www.metacartel.org/ (Retrieved 17/ 08/2020).

${ }^{79}$ Block.one Is a Leader in Providing High-Performance Blockchain Solutions. Available at: https://block.one/ (Retrieved 24/07/2020).
} 
stakeholders (Ølnes et al., 2017), the blockchain community advances several technical aspects of underlying technologies.

\section{Transaction Speed and Fees}

As a victim of its unexpected popularity, severe deficiencies in scalability of the original peer-to-peer concept of Bitcoin-derived blockchains based on PoW have surfaced. Transaction throughput still dwells many orders of magnitudes below established systems, for example, for facilitating payments with common credit card systems, albeit still generally faster than conventional banking; the limited bandwidth also led to soaring, occasionally even business impeding utility fees, for example, for "gas" on the Ethereum blockchain powering the majority of DeFi.

Strategies such as increased block sizes (Edwood, 2020) ${ }^{80,81}$ as well as refinements like "sharding" (Mearian, 2020), side chains (Singh et al., 2020), "ZK rollups" (Wu, 2019; ZeroKnowledge, 2020), recursive internetwork architecture (Cardano), and the "lightning network," 82 which resort to mechanisms like transient forking or off-chain processing, are currently elaborated and integrated into different blockchains. With their upgrade to version 2.0 expected for 2021 (O'Neal, 2020), the impactful Ethereum blockchain plans to transition from PoW to PoS to effectively tackle problems of transaction throughput and fees.

\section{Data Management}

As designed for the rather small information affiliated with financial transactions, the blockchain data structure is not well suited for storing large files. Indeed, it would be orders of magnitude more expensive to store a given amount of data on blockchain's ledger file distributed on a peer-to-peer network than on a single computer. Decentralized architectures (Benet 2015) ${ }^{83-85}$ and certain access protocols (Grishin et al., 2019) ${ }^{86}$ have been paired with blockchain to provide untampered data sets. A common method is to generate a cryptographic hash, that is, a unique, short, fixed-size fingerprint for a large data file which sensitively changes by even the slightest modification. Only this brief hash and a link to the file are stored on the blockchain. Perspectives for blockchain-based data governance have been conceived (Liu et al., 2019).

Also, the ingestion of external data into the system in a way that provides a high degree of data integrity poses a technical challenge. And even if it can be proven that data have not been

\footnotetext{
${ }^{80}$ Bitcoin Cash-Peer-To-Peer Electronic Cash. Available at: https://www. bitcoincash.org/ (Retrieved 02/09/2020).

${ }^{81}$ Bitcoin SV (Satoshi Vision). Available at: https://bitcoinsv.io/ (Retrieved 02/ 09/2020).

${ }^{82}$ Lightning Network-Scalable, Instant Bitcoin/Blockchain Transactions. Available at: https://lightning.network/ (Retrieved 24/07/2020).

${ }^{83}$ Filecoin. Available at: https://filecoin.io/ (Retrieved 07/09/2020).

${ }^{84}$ BigchainDB - The Blockchain Database. Available at: https://www.bigchaindb. com/ (Retrieved 11/09/2020).

${ }^{85}$ IPFS Powers the Distributed Web. Available at: https://ipfs.io/.

${ }^{86}$ OCEAN-A Decentralized Data Exchange Protocol to Unlock Data for AI. Available at: https://oceanprotocol.com/ (Retrieved 24/07/2020).
}

manipulated before entry, identifying the relationship between the transacting author and the data might not be completely automatable, although processes and protocols emerge, that might provide a foundation for automated and decentral rights management (Posth and Wittek, 2020).

\section{Interoperability, Configurability, and Sustainability}

There is a vast "zoo" of blockchain technologies and cryptocurrencies. Even the most established ones hardly communicate with each other. Large initiatives have formed toward building an "Internet of blockchains" ${ }^{87}$ which aims to establish standards and interoperability (Mapperson, 2020) of private and permissionless (public) blockchain protocols (IBC) as well as decentralized mechanisms for interchain exchange of crypto assets, for example, atomic swaps (BTC, 2020) or DEX (DEX). Similar to tool boxes for setting up web pages on classical internet, there are also initiatives to support rapid configuration of modular, opensource blockchains (Blenkinsop, 2019). ${ }^{88}$

With the skyrocketing popularity of ICOs, for example, during their short-lived hype in 2017, the issue of sustainability of blockchains arose, that is, what happens to the blockchain after the initial amount of funding has been spent, or its leaders lose interest and walk away. Among several constructs, treasury models which lock funds into smart contracts that are curated by stakeholders have been introduced to provide for continuous support and improvement (Cardano).

\section{Power Consumption}

The substantial environmental footprint required to entertain the trust-constituting computing power as well as the tendency toward dominating the hash rate by an oligopoly of miners through such PoW is deemed a major problem by many interest groups. More flexible cryptographic challenges that are unfavorable for ASIC mining and/or consume much less energy, such as (delegated) PoS (O’Neal 2020) ${ }^{89-91}$ protocols, have been implemented on various other blockchains in the meantime. Alternatively, widely trusted institutions like universities may partner to form a small network of nodes in a public-permissioned blockchain. In proof-of-authority (PoA)-based consensus formation, ${ }^{92}$ the consortium members vouch their valuable real-world reputation, rather

\footnotetext{
${ }^{87}$ Inter-Blockchain Communication (IBC)-The Interoperability Protocol Connecting the Global Economy to Blockchain Technology. Available at: https://cosmos.network/ibc (Retrieved 17/08/2020).

${ }^{88}$ Nuls. Available at: https://www.nuls.io/. (Retrieved 07/09/2020).

${ }^{89} \mathrm{DPoS}-$ Delegated Proof of Stake. Available at: https://en.bitcoinwiki.org/wiki/ DPoS (Retrieved 24/07/2020).

${ }^{90}$ Ouroboros-An Environmentally Sustainable, Verifiably Secure Proof-Of-Stake Protocol with Rigorous Security Guarantees. Available at: https://cardano.org/ ouroboros/ (Retrieved 07/09/2020).

${ }^{91}$ Fantom. Available at: https://www.fantom.foundation/ (Retrieved 07/09/2020).

${ }^{92}$ Bloxberg: Blockchain Infrastructure for Scientific Research. Available at: https:// bloxberg.org/ (Retrieved 24/07/2020).
} 
than expensive computing power (PoW) or crypto assets ( $\mathrm{PoS})$, to underpin the trust and integrity in the blockchain. With the release of its "Beacon Chain" (Shevchenko, 2020) in its 2.0 upgrade in December 2020, Ethereum, the second-largest blockchain by market cap, is gradually replacing PoW with a sophisticated, PoS-based consensus mechanism (among other improvements, e.g., regarding scalability) (Shevchenko, 2020). PoA or PoS consensus finding might be preferrable for blockchain-based projects in the scientific/academic community.

\section{Participation Models in Blockchains}

The organizational properties of a blockchain fall into essentially two broad categories: public or private and permissioned or permissionless (Sharma, 2019). The decentralized ledgers of Bitcoin (Nakamoto, 2009) or Ethereum (Buterin, 2014) are purposely permissionless, open and public, implying that there is no central authority restricting access and participation in the consensus mechanism, thus providing trust and neutrality while eliminating censorship and regulation across global communities. On the contrary, private or permissioned blockchains (Singh, 2019) restrict different levels of access to the ledger file and participation in the consensus mechanism; they have been devised to address concerns regarding, for example, performance, ownership, confidentiality, privacy, security, control, governance, scalability, configurability, consensus finding, and environmental footprint.

Such federated or consortium blockchains are well established in the corporate world (IBM, 2020), ${ }^{93-95}$ but are perceived controversial by larger parts of the crypto-community. They tend to consider these permissioned setups as a variant of a conventional corporate database where a central authority tasks verifiers and authorizers, thus lacking major features in terms of securing trust and protection against vulnerability, compared to their public counterparts.

\section{Organization and Governance}

The term "DAO" is not clearly defined (yet). Its incorporation might not bode well with the local legislative framework and entail huge risks for its (identifiable) stakeholders, for example, regarding liability. Possible advancements are distributed autonomous associations (DAAs), a checks and balances system, where individual roles are replaced by expert panels to disperse power from single points of failure. Suitable decision-making processes need to be clearly defined, for example, consensus-driven liquid democracy governance delegating day-to-day business to their appointed managers who periodically report and seek approval on business-critical or strategic issues.

\footnotetext{
${ }^{93}$ R3's Corda. Available at: http://www.r3.com (Retrieved 20/07/2020).

${ }^{94}$ Hyperledger. Available at: https://www.hyperledger.org/ (Retrieved 24/07/2020).

${ }^{95}$ B3i. Available at: https://b3i.tech/ (Retrieved 25/08/2020).
}

\section{APPLICATION TO SCIENCE AND RESEARCH, AND TECHNOLOGY DEVELOPMENT}

The spirit at the heart of blockchain may be articulated as finding trust and consensus within a noncustodial network of independent, potentially even malicious players; the integrity of the blockchain is fortified by a transparent, reward-based competition which demands risking some form of collateral from participants, for example, by investing in costly installation and operation of cutting-edge mining infrastructure.

Smart contracts and DApps have significantly extended the scope of applications that can be addressed by blockchain technologies; staking of crypto-assets and formalized reputation incentivize credible reporting of best possible truth to blockchain oracles and prediction markets, to optimize decision-making on allocation of resources toward achieving common project goals. Smart contracts have also enabled new asset types such as NFTs, ${ }^{96}$ and sophisticated manifestations of self-governance by liquid democracy (Liquid Democracy) and DAOs (DAO). First initiatives for providing a digital ecosystem to support collaboration on innovation processes through blockchain mechanisms have already surfaced (Rossum, 2017; Brock, 2018; Janowicz et al., 2018; van Rossum and Lawlor, 2018; Heaven, 2019; Bartling, 2020). ${ }^{97}$

Similarly, the research community rewards scientific discovery and technology development as well as finding consensus on their validation to eventually add new "blocks" of community-verified knowledge to a public, "ledger-equivalent" library that is captured in widely accessible, perpetual archives (Ducrée, 2020). Strong trends toward self-organization, autonomy of decision-making, for example, through peer review of publications and funding proposals, and even decentralization and competitive parallelization have always been a principal element of academic research.

Yet, the ranking and reward systems applying to researchers and institutions as well as career promotions, appointments to committees and invitations for high-caliber talks is not so rarely based on hidden rule sets and backroom arrangements. The valuation of intellectual assets, such as publications, inventions, and awards, remains somewhat obscure and inconsistent. Importantly, the community aspect of achievements is often poorly captured and artificially restricted to small groups, rather than acknowledging and thus incentivizing proactive engagement and commitment of lowerranked group members, peers, referees, and even noninstitutionalized individuals. Greater involvement of the community, for example, through hackathons and bounties, would certainly facilitate the formation of a critical mass of users, developers, and promoters, and thus energize fly-wheel effects to increase footprint and sustainability of projects.

\footnotetext{
${ }^{96}$ Non-fungible Token. Available at: https://en.wikipedia.org/wiki/Non-fungible_ token (Retrieved 04/01/2021).

${ }^{97}$ DEIP.world-Open Innovation Network. Available at: https://deip.world/ (Retrieved 24/07/2020).
} 
This section sketches the future scenario of a more inclusive, community-based approach toward science and RTD organized as a sophisticated betting game; blockchain orchestrates an arsenal of crypto-economic instruments for finding trust and best-possible truth at maximum transparency and minimum administrative costs for planning, funding, execution, management, assessment, arbitration, and exploitation of science and RTD projects (Figure 2). With some adequate modifications respecting the rule set and workflows of the corporate sector, the opportunity to flexibly tap into the increasingly broad pool of talent, expertize, creativity, labor, and facilities within a global village, rather than limiting contributions to centralized RTD headquarters, might largely outweigh reservations, for example, regarding ownership and protection of IP.

\section{Specifics of Science and Research, and Technology Development}

Investing in science and RTD is tied to a certain expectation toward the future, for example, to address a material need or find the answer to an important issue or question in society. In a legacy approach, a rather small cohort of experts is consulted to identify relevant topics, evaluate proposals, validate data, and assess outcomes.

Blockchain is so far mainly driven by software development for improving its own ecosystem and for its reach into new application spaces like industrial or food supply chains (Francisco and Swanson 2018; Wolfson, 2020), ${ }^{98}$ authenticity of precious commodities (Gokce et al., 2018; Alzahrani and Bulusu 2019; Javed, 2019), and enterprise solutions (IBM, 2020). ${ }^{93}$ Blockchain technologies also empower DeFi by creating novel financial instruments and investment vehicles such as yield farming (Yield Farming) for scooping arbitrage gains, synthetic assets (Synthetix), barrier-free, financially inclusive (micro-)payment systems (Ripple), DEX ${ }^{99,100}$ with AMMs, and services for auditing or insuring against crypto-specific risks. ${ }^{36}$ While making smaller economic impact, also significant work on Commons led by social objectives, to illuminate information processing (Alencar, 2020) and to support charitable initiatives, has been carried out (Ducrée et al., 2020). ${ }^{101}$

Somewhat on the contrary, most members of the science and RTD community, whether on the payroll of academia or industry, are primarily motivated by creation of knowledge; in addition to bonuses or share options in industry, their commitment and devotion is stimulated by recognition, respect, attention, prestige, individual fulfilment, funding, personal career opportunity, selffulfillment, joy of creativity, identification with their organization, utility to users and society, altruism, and, last but not least, having a saying in terms of management, refereeing or governance, and

\footnotetext{
${ }^{98}$ Cardano. Available at: https://cardano.org/ (Retrieved 03/09/2020).

${ }^{99}$ Decentralized Exchanges. Available at: https://defiprime.com/exchanges (Retrieved 20/08/2020).

${ }^{100}$ DEX.AG. Available at: https://dex.ag/ (Retrieved 20/08/2020).

${ }^{101}$ SEEDS-A Payment Platform and Financial Ecosystem to Empower Humanity and Heal Our Planet. Available at: https://joinseeds.com/ (Retrieved 24/07/2020).
}

opportunity for promoting a topic they find important in front of key stakeholders and large audiences.

As outlined in the preceding sections, the token economy that can be quite uniquely staged by blockchain-enabled reputation systems (Reputation System) is able to cater for objectively awarding and safely recording such personal achievements, stake them as collateral for quality and credibility of contributions, and to improve prediction markets. Hence, we propose here for the first time a token-economy as the basis to garner valuable community engagement for delivering topquality, well-reproducible and high-impact science and RTD.

This cooperative approach reflects characteristic features of the scientific community, such as self-organization, autonomy, open competition, decentralization, and transparency, to set a solid foundation for establishing trust, credibility, and objectivity in finding best-possible "truth." Especially the clear trend toward virtualization, for example, digital twin approaches, opens novel avenues toward widening participation of a global crowd to value creation, even to a novel type of freelance researcher, for example, by contributing intriguing hypotheses, novel concepts, improved modeling, simulation, and validation services.

\section{Science and Research, and Technology Development-Through the Eyes of Blockchain}

Along Figure 3, we illustrate the manifold similarities between the fabric of blockchain and the mechanisms underpinning the classic community of science and RTD. In DLT, transaction data are periodically collated, mined in an open cryptographic race (in public PoW networks), collectively verified, and selected according to predefined metrics and algorithms for amending a block to the existing chain. Crypto-economical mechanisms incentivize crowd participation and create trust.

On a conceptual level, there are remarkable similarities of blockchain to the modus operandi of the scientific community. Scientific discoveries may, for instance, be expressed by a hypothesis that is embedded into a context, described by a model of understanding, verified by established experimental protocols and methods of data analysis. These "blocks" representing scientific discoveries, research data, or technological inventions are then released to the scientific community for assessment and validation by peers according to widely accepted criteria and methods that tend to be specific to each research discipline (Reyes et al., 2021).

To optimize quality, and to avoid manipulation and scamming, authors stake their reputation (PoS). Upon approval, the blocks are appended to the "ledger of knowledge" that is archived in various forms, for example, publicly in journal publications or patents, or in internal documents stored on institutional or corporate databases. In the absence of immediate consensus and analogously to "reorgs" and "forks" in blockchain, various theories ("chains") transiently compete until sufficient evidence ("blocks") has been aggregated to convince a critical mass of the community.

Having identified these analogies to protocols in blockchain, we notice that involvement of the scientific community is highly relevant to reviewing, but contributions of the crowd at large to the creation of initial IP, validation, and dissemination are rather poorly incentivized 


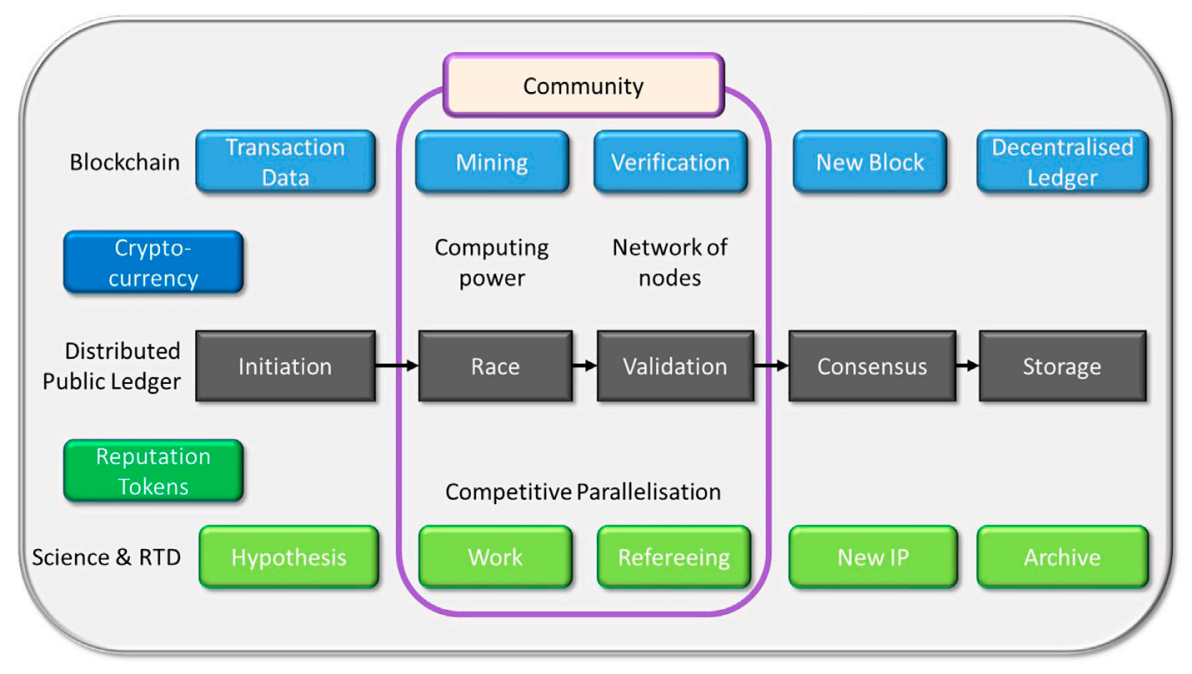

FIGURE 3 | Analogies between science and RTD and the distributed ledger technologies (based on PoW). Permissionless blockchains, such as Bitcoin or Ethereum, can be interpreted as cooperative peer-to-peer networks of independent players for growing a record of transactions that are bundled in consecutive blocks. These packets of transaction data are competitively and redundantly created, time-stamped, verified, and selected for inclusion according to a deterministic, codebased consensus protocol. Staking PoW through participation in an enormous group mining effort, reputation by PoS and utility costs mitigate spamming and reward contributions to securing the integrity of the decentralized, non-repudiable ledger. "Hashing," for example, by Merkle trees, intertwines new with existing blocks to firmly secure the blockchain. The "blocks" in science and RTD may be IP documented by articles, internal reports, or patent fillings that describe new knowledge or knowhow that has been reviewed by peers in the community. Trust and transparency are afforded by referencing and working according to established, collectively accepted methods. This IP is captured, for example, as non-fungible tokens (NFTs), on public or private, quasi-eternal, and unforgeable archives curated by trusted journals, publishing houses, or patent offices. Authors, inventors, validators, and referees engage in a "race" to be first to add high-impact and well-reproducible results to this public "ledger of knowledge." Their successful efforts may be rewarded by direct monetary benefits, but often even stronger incentivized by career options, citations, and overall recognition/prestige of the community. Spamming of the ledger with "junk" is averted by peer review and risking reputation. Both systems prove to autonomously function without central authority to certify the integrity of data blocks and knowledge, respectively.

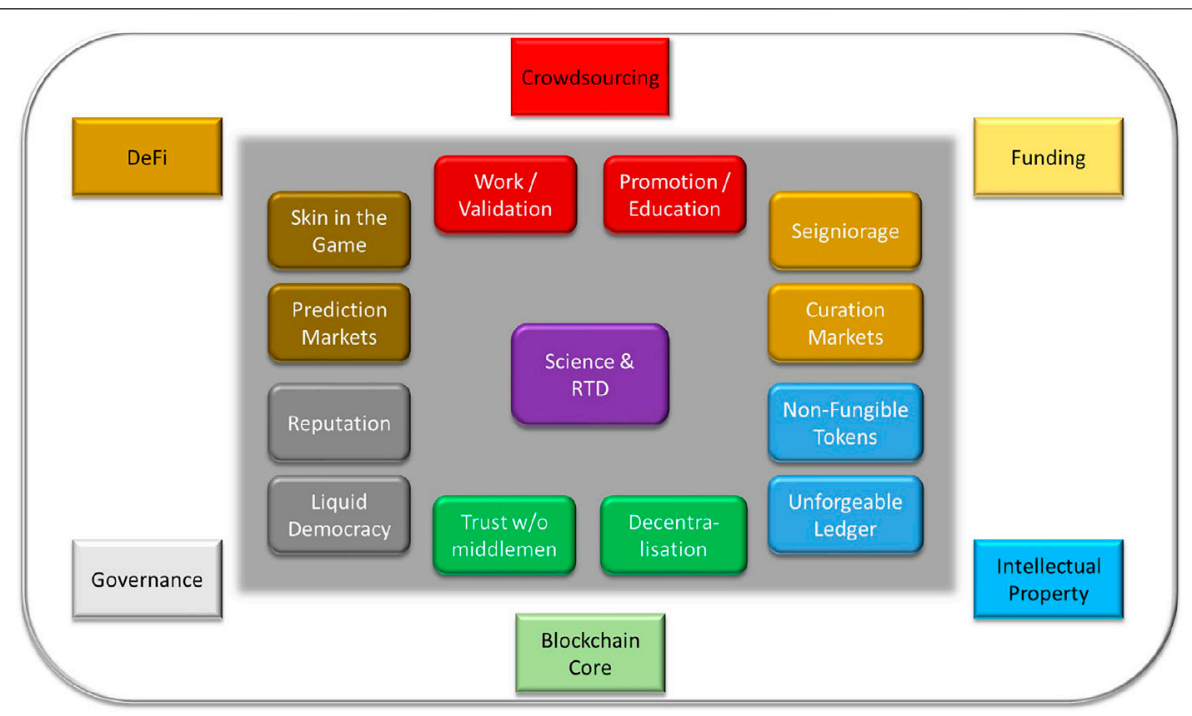

FIGURE 4 | Innovation potential for science and RTD projects through crypto-economically incentivized involvement of collective intelligence. Core blockchain technologies provide trust and trust-finding mechanisms without requiring a central authority. Smart contracts enable skin-in-the-game staked prediction markets, novel, liquid democracy style governance schemes involving formalized reputation, a tamper-proof and time-stamped ledger to register intellectual property (IP) which may be represented and traded as non-fungible token (NFT). In addition to conventional mechanisms, project funding can arise from seigniorage and curation markets. The blockchain-backed tokenization can thus effectively crowdsource project contributions, for example, work, validation, promotion and education, collective intelligence through remuneration, and reputation tokens to thus optimize the project outcomes while acknowledging contributions from individual stakeholders. 
and acknowledged (Figure 3). The following sections describe how a blockchain-based token economy highly encourages a collaborative approach for the mutual benefit of contributors and the objectives of the project, as illustrated in Figure 4.

\section{Tokens}

Blockchain technologies can uniquely implement transparent rule sets on its tamper-free and immutable ledger to incentivize, credit, and reward a broad repertoire of community contributions. We first illustrate the benefits such a token economy can offer for science and RTD projects (Figure 4). Evidently, seigniorage (Seignorage), ICOs, ITOs, STOs, and dynamically evolving crowdfunding (Edmondson, $2020)^{13,74,103}$ can channel financial resources at different stages of progression into a project. These assets may be used to directly recruit, crowdsource, and incentivize workforce, idea creation, contributions of expertize, management, and other services.

Historically, free-market economies, potentially padded with rule sets on anti-trust to avoid monopolies and social aspects, have proven to outperform centralized, state-directed economies, despite potential duplication and friction losses between competitors. In the same way, such "tokenization" can drive a healthy rivalry, also termed "competitive parallelization" (Ducrée et al., 2020a), toward performance enhancement of the full gamut of project contributions, whether internally or externally crowdsourced (Ducrée, 2019), thus optimizing the overall outcome of science and RTD projects.

In reputation systems, ${ }^{91,103-105}$ tokens are earned or burned, depending on the best correlation between predictions and target outcomes (Numerai). Skin in the game schemes can effectively "dilute" compromised assessment, for example, by malicious participants setting up numerous pseudonymous identities and using them to gain a disproportionately large influence. Mechanisms for counteracting such "Sybil attacks", 106 for example, through "proof of identity," have been elaborated (Kurve and Kesidis, 2011; Bansal and Misra, 2016). Reputation tokens can mirror track record, for example, obtained for quality and speed of project work, validation, advise, education, team building, promotion, and leadership (Cant), to substantiate the overall credibility of contributors. In contrast with the current state where even the number of citations of a particular paper or author is usually only available under subscription models, blockchain implementation would offer rendering such records publicly available.

Similar to artwork or collectibles (Posth, 2020), ${ }^{61}$ NFTs can issue a legally valid time-stamped proof of knowledge of intellectual assets, for example, by provision of hypotheses,

\footnotetext{
${ }^{102}$ Indiegogo-Get the Tech that Gets People Talking. Available at: https://www. indiegogo.com/ (Retrieved 18/08/2020).

${ }^{103}$ Reputation System. Available at: https://en.wikipedia.org/wiki/Reputation_ system (Retrieved 28/08/2020).

${ }^{104}$ DREP. Available at: https://www.drep.org/ (Retrieved 07/09/2020).

${ }^{105}$ ONTology-Trust Redefined. Available at: https://ont.io/ (Retrieved 07/ 09/2020).

${ }^{106}$ ARTiFACTS-Researcher Recognition. Available at: https://artifacts.ai/ (Retrieved 24/07/2020).
}

methodology, experimental design, theoretical framework, supporting data set, and statistical interpretation, to indisputably claim inventorship to be recorded on patent applications or authorship on a scientific discovery. Portals already exist to capture and manage access to such $\mathrm{IP}^{89,103}$ and data (Grishin et al., 2019). ${ }^{86,92,107-109}$

In addition, many existing implementations rely on asset identification based on cryptographic hashes to act as generic signatures of data. Although they are well suited to uniquely identify a certain data instance, their lack of locality-preserving and perceptual hashing properties seriously limits application in the copyright and patent domain, especially considering automated processing. In order to unlock the full potential of this technology, alternative fingerprinting algorithms like International Standard Content Code (ISCC) (Titusz and Niederbühl, 2020) need to be explored. As patent and copyright law are intrinsically complex, the process involved might not be completely automatable; different instruments, such as the Open Content Certification Protocol (OCCP) (Posth and Wittek, 2021), aim to provide a foundation for machine-to-machine communication and technology-based trust, while still allowing for a soft factor of human assessment to be injected into the system.

Commercially relevant IP represented by NFTs might also be better tradable through tokens, especially for academic institutions whose technology transfer offices (TTOs) are usually netting significant losses. Issues around regulation and confidentiality might be addressed by permissioned blockchains (Singh). To embed this novel mechanism into a legally sound framework, buyin from patent offices is still to be pushed on the political level.

Even beyond such core innovative contributions, the entire team might be further incentivized by (potentially paninstitutional) reputation tokens, for example, obtained for work, validation, advise, education, team building, leadership, and promotion (Cant, 2019). Smart contracts can also time-lock tokens, that is, their "pay out" might be linked to sustainable outcomes that are evaluated as results have created measurable impact, rather than encouraging a transient flash in the pan.

Toward mustering a critical mass, tokens can also be issued to (external) users for popularizing the project (Leshner, 2020), for example, through referrals and postings, or by proof of "attention," for example, through completing specific tutorials and browsing activities. ${ }^{110,111}$ This, at first sight, altruistic giveaway approach makes better sense if promotion is considered as instrumental for engaging a critical number of engaged users. In a certain way, researchers nowadays already accept similar marketing expenses, for example, in the form of registration fees for presenting their results at conferences or for

\footnotetext{
${ }^{107}$ Nebula Genomics. Available at: https://nebula.org/ (Retrieved 23/09/2020).

${ }^{108}$ Oasis Labs-Unlock Your Most Valuable Data. Available at: https://www. oasislabs.com/. (Retrieved 24/07/2020).

${ }^{109}$ ScienceMatters-The Next-Generation Science Publishing Platform. Available at: https://www.sciencematters.io/ (Retrieved 23/09/2020).

${ }^{110}$ BAT-Basic Attention Token. Available at: https://basicattentiontoken.org/ (Retrieved 17/08/2020).

${ }^{111}$ Brave Browser. Available at: https://brave.com/ (Retrieved 17/08/2020).
} 
publishing open-access articles to optimize their reach and future citation counts. Furthermore, tokens might be passed out as "social signal" to teams in the community that follow similar research goals or ethical values, ${ }^{75}$ thus creating momentum in community building.

\section{Benefits for Token Holders}

Tokens may be deemed digital assets which may be swapped into alternative crypto-assets at secondary markets, converted into fiat currencies at various exchanges, be staked, simply "hodled", 18 or spent on settling utility fees for transactions on the blockchain. Tokens can also provide credibility for increasing the weight of a user's saying in the project ecosystem, for example, on blockchain-supported, liquiddemocracy equivalents to traditional governance, scientific advisory boards or arbitration panels, or a DAO where, in its purest implementation, most decisions need clearance by (token-weight) vote of their stakeholders.

Also, the project itself might establish a rule set which tags an internal value to the tokens. ${ }^{112}$ In addition to project ownership associated with participation in value creation (dividends) and project direction (management), blockchain-recorded personalized tokens can account for credible certification of soft skills, professional and academic qualifications Rana (2020), or hard facts like authorship, impact factors, research income, and invention disclosures, for example, to enhance CVs or for boosting job applications at different (participating) organizations. Here, we can envision a conceptual and technological alignment with the domain of self-sovereign identity (SSI), such as the Verifiable Credentials Data Model, developed by the W3C (Sporny et al., 2019). Other tokens may be accrued by team members to avail of popular incentives, for example, career promotion, bonus payments, additional holidays, travel upgrades, a company car, or be pooled for sponsoring team events.

\section{Token-Staked Prediction Markets}

Further to the previously quoted benefits, tokens can be used as "skin in the game" to foster community approaches for optimized assessment, planning, and forecasting project uncertainties. In fact, broad involvement of expertize, skill, and labor in generating knowledge has always resided at the very core of science and RTD, and mostly administrative hurdles have confined outreach on a broader scale. At least in principle, these barriers, which are somewhat hampering progress and consensus on extending the "knowledge", may be lifted by adopting previously described liquid democracy strategies, and staking mechanisms, such as PoW or PoS, that are already underpinning blockchain.

More specifically, prediction markets can be created where all interested parties, whether workers and investors as well as project-internal and external experts and evaluators, can stake their tokens to support their credibility for contributing, assessing, and future-proofing the project, thus creating synergistical win-win scenarios. Curation

${ }^{112}$ COLONY.io-Organizations, for the Internet. Available at: https://colony.io/ (Retrieved 24/07/2020) markets (de la Rouviere, 2017; Rouviere, 2017) and TBCs (Goro, 2018) can be set up very swiftly on blockchain to receive valuable feedback, publicity and engagement on early-stage project ideas, their implementation plans and first results. In their endgame, and to differentiate them from ICOs that may be subject to rather strict financial legislation, contributors from the scientific community might be rewarded by formalized reputation tokens which acknowledge their capability to identify the potential while the project still dwells at its infant stage; the tokens earned this way can be used in other projects, for example, to increase voting power on governance and arbitration boards. TBC protocols might also incorporate mechanisms for (partially) funding the projects, for example, through arbitrage from trading tokens or providing liquidity to secondary markets.

While there is still some pioneering work to be carried out for conclusively demonstrating that non-fiscal instruments enhance a business proposal toward investors, for example, in the form of an ICO or DAO, tokens awarded for intellectual achievements would certainly epitomize a not (directly) monetizable value that most researchers would still be eager to accumulate and utilize for their interests.

\section{Publication, Peer Review, and Funding System}

In the first half of the 20th century, scientific journals started filtering submissions through critical assessment by independent colleagues. This peer-review system (Baldwin, 2018) has essentially survived into modern days, in some fields supplemented by electronic preprint servers ${ }^{113,114}$ which release manuscripts prior to full submission, usually to enquire constructive upfront feedback from colleagues in the community, and to establish precedence of the work. While the system of scientific publishing has proven to function for the most part, it is, at least at times, still prone to poor quality and even nepotism, for example, due to paucity of highly qualified referees caused by poor incentivization, the once-off nature and anonymity of reviewer involvement, substandard publishing houses, and the practical absence of rewards and penalties for poor judgment on project impact.

Blockchain technologies could also provide fair models for access to scientific data. In its current state, large portions of the scientific record are siloed in private enterprise or only available through pricy subscription models, including important metrics such as citation numbers.

It is evident that the collateral-backed reputation systems like token economies and prediction markets coordinated by blockchain's decentralized and transparent peer-to-peer network would be wellsuited to boost leverage of crowd intelligence, thus enhancing quality, credibility, reproducibility, and impact of findings, and, importantly, their broad-scale adoption in the community. These shortcomings affect publications as well as typical funding mechanisms

\footnotetext{
${ }^{113}$ arXiv. Available at: https://arxiv.org/ (Retrieved 04/01/2021).

${ }^{114}$ medRxiv-The Preprint Server for Health Sciences (Retrieved 10/08/2020).
} 
implemented by public agencies, where blockchain-based staking of reputation and crypto assets could help formulate calls and support scientific progress, monitor administration, and exploitation.

However, note that the publishing based on blockchain technology and within its community, with exceptions (Rossum, 2017, Janowicz et al., 2018, Martin, 2018, van Rossum and Lawlor, 2018, Dhillon, 2019, Heaven, 2019, Leible et al., 2019, Mackey et al., 2019, Rafati Niya et al., 2019), ${ }^{98,107,110,116-118}$ is so far dominated by unaudited white papers, non-systematically archived web pages, unfiltered news releases, and hardly traceable social media contributions which are commented on rather secluded Internet forums. This rather Bohemian publishing culture, and a stark clash of jargon, contrasts the traditional path for publishing in the scientific community, which involves vigorous peer review for affording credibility and quality and reputed publishers for assuring documentation that is stable on the long term.

\section{Decentralization}

Depending on the objective of the blockchain, different organizational setups may be chosen for the blockchain technology. Commercial RTD projects might want to deliberately restrict access in permissioned, corporate/consortium/federated blockchains, for example, to guarantee confidentiality and retain full control of blockchain operations, and for lowering demands on computing infrastructure and their environmental footprint. On the contrary, academically minded scientific projects, for example, for creating fundamental knowledge, might want to be more transparent, thus suggesting implementation on a permissionless or permissioned public blockchain.

As the visionary pinnacle of transformation, science, and RTD could be managed by DAOs, which assume the function of publishing and funding including review, monitoring, and impact creation. This would actually well comply with the century-old aspiration of research toward autonomous self-administration in a decentralized and objective approach of the global community, which is driven by the productive interplay of healthy competition and self-interest to efficiently pursue Commons (Ducrée et al., 2020a) for the good of people, societies, and economies.

The management structure of DAOs would be transparently coded into blockchain-engraved algorithms which can be complemented by arbitration schemes, and upgraded by liquid democracy governance, as already executed in existing blockchain projects. ${ }^{54,55}$ By offering a broad range of involvement and commitment staked by reputation and cryptoassets, pivotal flywheel effects can be unleashed toward creating a critical mass of developers, early adopters, and highly engaged users, which, in turn, would generate significant pressure on

\footnotetext{
${ }^{115} \mathrm{ORCiD}$-Connecting Research and Researchers. Available at: https://orcid.org/ (Retrieved 22/09/2020)

${ }^{116}$ Orvium-Accelerating Scientific Publishing. Available at: https://orvium.io/ (Retrieved 22/09/2020).

${ }^{117}$ DECENT. Available at: https://decent.ch/ (Retrieved 23/09/2020).

${ }^{118}$ ResearchHub. Available at: https://www.researchhub.com (Retrieved 19/ $11 / 2020)$.
}

legislators to accommodate and embrace the great promise of blockchain technology for science and RTD.

\section{Nucleation of Projects}

While the advantages of a blockchain-based implementation have been highlighted, the challenge will be to graft project funding onto present, agency-based architectures, as well as to create a critical mass of community involvement. To start out, a "Minimum Viable Product/Project" (MVP) needs to be defined, which may be a scaled-down version featuring only select aspects of the bigger picture sketched in this work. Figure 5 suggests a possible, dualtrack strategy for projects to tap into conventional public or corporate funding streams, thus catering for real-world expenses to be reimbursed in fiat money, while entertaining a crypto-economically incentivized "betting" game for effectively involving the wisdom of the crowd way beyond legacy project structures.

In a subsequent stage, the project itself, including the left-hand part of Figure 5, could be managed through blockchain technologies, for example, by operating with fiat-pegged stablecoins for conventional financial transfers. In this interim model, certain fiat could be converted into cryptocurrencies for increasing the incentives fueling the modules right-hand side. Transparent, multi-signature fund management and IP protection incentivize individuals to contribute financially and intellectually.

In a decentralized future scenario, even the source of fiat, for example, a public agency, foundation, or corporation, could be replaced by open crowdfunding, to ultimately integrate the entire organizational structure, encompassing publishing and exploitation, into a smart-contract-encoded DAO that relies on PoS-linked skin in the game within a full-fledged crypto-economical ecosystem.

\section{SUMMARY, CONCLUSIONS, AND OUTLOOK}

We have surveyed key instruments of blockchain-enabled token economies that already underpin core elements of their ecosystem and a range of important application spaces such as store of value (mainly Bitcoin), decentralized finance (DeFi), and commercial supply chains as well as certification of provenance and ownership of unique goods like property and artwork (NFTs).

We have argued that powerful reputation systems that are enabled by blockchain's smart-contract-supported crypto-economy can seminally augment the traditional processes in science and RTD to advance to a globally inclusive "Internet of knowledge" (Ducrée, 2020). Incentivized by such crypto assets that are awarded for a wide range of project contributions like idea creation, planning, assessment, funding, work, interpretation, validation, tutoring, leadership, team building, management, marketing and exploitation, collective intelligence, skills, and infrastructure can be efficiently crowdsourced for projects, whether in academic research, corporate consortia, or grassroots initiatives.

Researchers can utilize these tokens to execute and possibly delegate their reputation-weighted voting rights in liquiddemocracy style governance, stake them in secondary prediction markets, or to support their proposals for funding. In a competitive, firmly community-anchored participatory research approach, the token economy can thus markedly enhance the quality, 


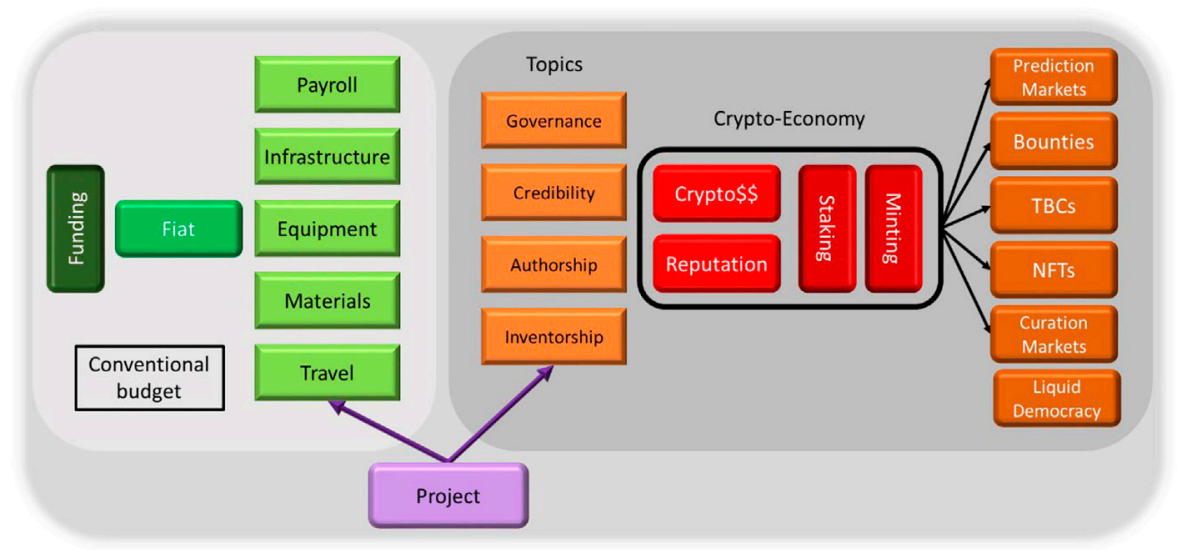

FIGURE 5 | Concept for grafting a blockchain-enabled project onto existing funding and budget schemes. In a two-pronged approach, real-world expenses and salaries are reimbursed by classical funding schemes in fiat money. Aspects of governance, credibility, author, and inventorship leverage a token economy revolving around minting and staking of tokens representing crypto-assets and reputation. This token economy offers novel instruments for project optimization such as prediction and curation markets, bounties rewarded for identifying or solving particular issues, token bonding curves (TBCs) to incentivize thorough assessment of early-stage ideas by skin-in-the-game, non-fungible tokens (NFTs), for example, for assigning contributions to IP and a liquid democracy-guided management structure. The portrayed strategy will seminally enhance project outcomes by comprehensive community involvement and increase motivation for contributors.

credibility, reproducibility, speed, transparency, cost-efficiency, and mission critical user engagement and adoption of project outcomes, and thus also decisively contribute to address the notorious reproducibility crisis of science.

By virtue of the outstanding importance of nonmonetary reputation rewards for motivating most researchers, regulatory restrictions are unlikely to impede implementation of the token economy in science and RTD. It is foreseeable that, in combination with cloud computing, global logistics and world-wide open-access facilities providing co-working spaces, equipment, training, and competent staff for making, characterizing, and developing "things"3,4 that significantly lower entry barriers and risks for research activities, the token economy will breed new types of freelance researchers, citizen scientists, garage entrepreneurs, and digital nomads, whose special expertize and work force are flexibly recruited into projects based on objectivized performance criteria rather than affiliation.

A trusted, blockchain-backed infrastructure that includes standards for token-backed funding, oracle services to prove the validity of the outcomes, and mechanisms for protecting ideas and assuring a favorable compensation to workers and quality to funders, for example, as validated by independent auditors and proper dispute resolution processes, can lead to a paradigm shift toward an open, community-based organization of science and RTD. Blockchainenabled decentralization thus nurtures the emergence of heterogeneity, of small, creative, and talented individuals or teams forming "solution centers" that can build up a reputation for efficiently solving a certain category of tasks while operating from anywhere on the planet. As elaborated in this work, the future of science and RTD will emerge on top of a new form of decentralized social networks.

Compelling advantages in trust, cost, and speed will substantially push acceptance of token economies; administrative barriers may be overcome if continued to be driven by major academic and commercial players and the financial sector. Also, the present dialog with governments, and public entities like land registries, patent offices, funding agencies, regulatory bodies, and central banks (Erazo, 2020;
Haig, 2020; Hashim et al., 2020; Partz, 2020; Post, 2020) 39,40,119-121 needs to be intensified to fully embrace the blockchain.

Yet, strong adherence to tradition presents a tall barrier for gathering critical momentum for major revision of the legacy schemes in science and RTD. There is a blatant gap in culture, for example, toward procedures for funding, publishing, documentation, reviewing, and auditing. In fact, even for composing this very article, many citations point to web sites hosting transient content and evading independent peer review; they are thus likely to be biased and marketing driven and less suitable for historical archiving than articles in traditional journal libraries. Furthermore, the still quite volatile environment of blockchain, with issues such as workflow compatibility, satisfactory user experience (UX), and understanding of the crypto-economy, needs to be convincingly resolved to efficiently promote its wide-spread adoption in science and RTD scene.

Realistically, transition to a crypto-economical approach by science and RTD communities would have to be gradual, and possibly seeded by a sizable crowd of benevolent early adopters; while the landslide proliferation of Bitcoin appears to prove the opposite, wide-scale adoption of the blockchain-founded concepts proposed here very likely needs to be fostered by well-funded campaigns to sensitize the community at large, for which the disruptive concepts of blockchain are still rather alien. Amara's law ${ }^{122}$ seems to well summarize the

\footnotetext{
${ }^{119} \mathrm{EU}$ Blockchain Infrastructure. Introducing the European Blockchain Services Infrastructure (EBSI). Available at: https://ec.europa.eu/cefdigital/wiki/display/ CEFDIGITAL/EBSI (Retrieved September 29, 2020).

${ }^{120}$ European Commission. Legal and Regulatory Framework for Blockchain. Available at: https://ec.europa.eu/digital-single-market/en/legal-andregulatoryframework-blockchain (Retrieved September 28, 2020).

${ }^{121}$ German Ministry of Finance Calls for Blockchain-Based, Digital, Programmable Euro. Available at: https://fintechnews.ch/blockchain_bitcoin/german-ministry-of-financecalls-for-blockchain-based-digital-programmable-euro/38081/ (Retrieved 26/08/2020).

${ }^{122}$ Roy Amara. Available at: https://en.wikipedia.org/wiki/Roy_Amara (Retrieved 17/08/2020).
} 
status quo and prospects for the opportunity for blockchain in science and RTD: "We tend to overestimate the effect of a technology in the short run and underestimate the effect in the long run."

\section{DATA AVAILABILITY STATEMENT}

The original contributions presented in the study are included in the article/Supplementary Material. Further inquiries can be directed to the corresponding author.

\section{REFERENCES}

Alencar, M. (2020). Generalized Token Curated Registries: The World of Lists. KLEROS, Available at: https://blog.kleros.io/generalized-token-curatedregistries/ (Accessed September 3, 2020).

Alzahrani, N., and Bulusu, N. (2019). A New Product Anti-counterfeiting Blockchain Using a Truly Decentralized Dynamic Consensus Protocol. Concurr. Comp. Pract. Exp. 32 (5), e5232. doi:10.1002/cpe.5232

Austin, S. (2020). Understanding the Landscape of Decentralized Finance. Available at: https://cointelegraph.com/news/understanding-the-landscapeof-decentralized-finance

Baker, M. (2016). 1,500 Scientists Lift the Lid on Reproducibility. Nature 533, 452-454. doi:10.1038/533452a

Baldwin, M. (2018). Scientific Autonomy, Public Accountability, and the Rise of "Peer Review" in the Cold War United States. Isis 109, 538-558. doi:10.1086/700070

Bansal, H., and Misra, M. (2016). "Sybil Detection in Online Social Networks (OSNs)," in Proceedings of the 2016 IEEE 6th International Conference on Advanced Computing (IACC), Bhimavaram, India, February 27-28, 2016, 111. doi:10.1109/IACC.2016.111

Becker, H. (2010). Collective Wisdom. Lab on a Chip 10, 1351-1354. doi:10.1039/ c004239k

Benet, J. (2015). IPFS-InterPlanetary File System. Available at: http://github.com/ ipfs/ipfs

Bartling, S. (2020). Blockchain for Science. Available at: https://www. blockchainforscience.com/ (Retrieved July 24, 2020)

Birch, J. (2019). Blockchain and 3D Printing Are Reinventing Aerospace Supply Chains. Available at: https://cointelegraph.com/news/blockchain-and-3dprinting-are-reinventing-aerospace-supply-chains

Blenkinsop, C. (2019). Open-Source Platform Lets Users Build Their Own Blockchain in Under $10 \mathrm{Min}$. Available at: https://cointelegraph.com/news/ open-source-platform-lets-users-build-their-own-blockchain-in-under-10-min

Blenkinsop, C. (2020). Q\&A: How Blockchain Could Transform the Art Industry. Available at: https://cointelegraph.com/news/qa-how-blockchain-couldtransform-the-art-industry

Brock, J. (2018). Could Blockchain Unblock Science? Available at: https://www. natureindex.com/news-blog/could-blockchain-unblock-science

BTC (2020). What Are Atomic Swaps? Available at: https://bitcoinmagazine.com/ guides/what-are-atomic-swaps. (Retrieved July 24 2020).

Buterin, V. (2014). White Paper. Ethereum/wiki Wiki - GitHub. Available at: https://github.com/ethereum/wiki/wiki/White-Paper

Cant, J. (2019). Enterprise Ethereum Alliance's Reward Token System Backed by Microsoft, Intel. Available at: https://cointelegraph.com/news/enterpriseethereum-alliances-reward-token-system-backed-by-microsoft-intel

Chen, T.-C. T. (2020). 3D Printing and Ubiquitous Manufacturing. Berlin, Germany: Springer. Available at: https://www.springer.com/gp/book/9783030491499.

Congdon, K. (2020). Inside Pfizer's Modular Manufacturing PODs. Available at: https://www.pharmaceuticalonline.com/doc/inside-pfizer-s-modular-manufacturingpods-0001

Dale, B., and Foxley, W. (2020). Story from Tech DeFi Leader MakerDAO Weighs Emergency Shutdown Following ETH Price Drop. Available at: https://www. coindesk.com/defi-leader-makerdao-weighs-emergency-shutdown-followingeth-price-drop

\section{AUTHOR CONTRIBUTIONS}

All authored reviewed the entire manuscript. JD developed the main concept and authored the full draft manuscript. RW, KW, AI, WP, DK, and JL mainly advised on blockchain technology; $\mathrm{TH}$ on open science and community-based participatory research; SP on NFTs, decentralization, and scarcity; ME and SB on blockchain for research; NW on the reproducibility crisis in science; and IP on circular economy and $\mathrm{TP}$ on publishing.

Daly, D. (2020). Salary \& Motivation, Do They Go Hand in Hand? Available at: https://payslip.com/blog/payroll-salary-and-motivation-do-they-go-handin-hand/

Davidov, I. (2019). The Crypto Betting Industry (Prediction Markets): An Overview. Available at: https://www.datadriveninvestor.com/2019/11/15/thecrypto-betting-industry-prediction-markets-an-overview/

de la Rouviere, S. (2017). Introducing Curation Markets: Trade Popularity of Memes \& Information (With Code)! Available at: https://medium.com/@ simondlr/introducing-curation-markets-trade-popularity-of-memes-informationwith-code-70bf6fed 9881

Detweiler, L. (2020). Sybil Attack. Available at: https://en.wikipedia.org/wiki/Sybil_ attack (Retrieved August 28, 2020)

Dhillon, V. (2019). Blockchain Based Peer-Review Interfaces for Digital Medicine. F. Block. 3, 8. doi:10.3389/fbloc.2020.00008

Ducrée, J. (2019). Efficient Development of Integrated Lab-On-A-Chip Systems Featuring Operational Robustness and Manufacturability. Micromachines 10, 886. doi:10.3390/mi10120886

Ducrée, J., Etzrodt, M., Gordijn, B., Gravitt, M., Bartling, S., Walshe, R., et al. (2020a). Blockchain for Organising Effective Grass-Roots Actions on a Global Commons: Saving the Planet. F. Block. 3, 33. doi:10.3389/fbloc.2020.00033

Ducrée, J., Gravitt, M., Walshe, R., Bartling, S., Etzrodt, M., and Harrington, T. (2020b). Open Platform Concept for Blockchain-Enabled Crowdsourcing of Technology Development and Supply Chains. Frontiers in Blockchain 3, 386525. doi:10.3389/fbloc.2020.586525

Ducrée, J. (2020). Research-A Blockchain of Knowledge? Blockchain - Research and Applications 1, 100005. doi:10.1016/j.bcra.2020.100005

Dwan, K., Altman, D. G., Arnaiz, J. A., Bloom, J., Chan, A. W., Cronin, E., et al. (2008). Systematic Review of the Empirical Evidence of Study Publication Bias and Outcome Reporting Bias. PLoS One 3, e3081. doi:10.1371/journal.pone. 0003081

Edmondson, B. (2020). How Bitcoin and Blockchain Are Changing Crowdfunding. The Balance 2020. Available at: https://www.thebalance.com/how-bitcoin-andblockchain-is-changing-crowdfunding- 4173837

Edwood, F. (2020). Block Size and Scalability, Explained. New York, NY: Cointelegraph. Available at: https://cointelegraph.com/explained/block-sizeand-scalability-explained

Entriken, W., Shirley, D., Evans, J., and Sachs, N. (2020). EIP-721: ERC-721 Nonfungible Token Standard. Ethereum Improvement Proposals. Available at: https://eips.ethereum.org/EIPS/eip-721

Erazo, F. (2020). World's First Crypto Bank Adds Support for Ripple's XRP. New York, NY: Cointelegraph. Available at: https://cointelegraph.com/news/worldsfirst-crypto-bank-adds-support-for-ripples-xrp

Foxley, W. (2021). 5 Years after Launch, Predictions Market Platform Augur Releases Version 2. New York, NY: Coindesk. Available at: https://www. coindesk.com/5-years-after-launch-predictions-market-platform-augur-releasesversion-2

Foxley, W. (2020). Everything You Ever Wanted to Know about the DeFi "Flash Loan" Attack. New York, NY: Coindesk. Available at: https://www. coindesk.com/everything-you-ever-wanted-to-know-about-the-defi-flashloan-attack

Francisco, K., and Swanson, D. (2018). The Supply Chain Has No Clothes: Technology Adoption of Blockchain for Supply Chain Transparency. Logistics 2, 2. doi:10.3390/logistics2010002 
Freedman, L. P., Cockburn, I. M., and Simcoe, T. S. (2015). The Economics of Reproducibility in Preclinical Research. PLoS Biol. 13, e1002165. doi:10.1371/ journal.pbio. 1002165

Gokce, O., Mercandetti, C., and Delamarche, E. (2018). High-Content Optical Codes for Protecting Rapid Diagnostic Tests from Counterfeiting. Analytical Chemistry 90, 7383-7390. doi:10.1021/acs.analchem.8b00826

Goro, J. (2018). Token Bonding Curves Explained. Medium.com 2020. Available at: https://medium.com/coinmonks/token-bonding-curves-explained-7a9332198e0e

Grieves, M., and Vickers, J. (2017). "Digital Twin: Mitigating Unpredictable, Undesirable Emergent Behavior in Complex Systems," in Transdisciplinary Perspectives on Complex Systems. Cham, Switzerland: Springer, 85-113. doi:10. 1007/978-3-319-38756-7_4

Grishin, D., Obbad, K., and Church, G. M. (2019). Data Privacy in the Age of Personal Genomics. Nat Biotechnol 37, 1115-1117. doi:10.1038/s41587-0190271-3

Haig, S. (2020). SEC Loosens Crowdfunding Guidelines amid Economic Slowdown. New York, NY: Cointelegraph. Available at: https://cointelegraph. com/news/sec-loosens-crowdfunding-guidelines-amid-economic-slowdown

Hashim, H. (2020). Federal Agencies Turning to Blockchain for its Benefits. New York, NY: Cointelegraph. Available at: https://cointelegraph.com/news/federalagencies-turning-to-blockchain-for-its-benefits

Head, M. L., Holman, L., Lanfear, R., Kahn, A. T., and Jennions, M. D. (2015). The Extent and Consequences of P-Hacking in Science. PLoS Biol. 13, e1002106. doi:10.1371/journal.pbio.1002106

Heaven, D. (2019). Bitcoin for the Biological Literature. Nature 566, 141-142. doi:10.1038/d41586-019-00447-9

Hill, S., and Ready-Campbell, N. (2011). Expert Stock Picker: The Wisdom of (Experts in) Crowds. Int. J. Electron. Commer. Stud. 15 (3), 73-102. doi:10.2753/ JEC1086-4415150304

Hodgson, M. (2016). A Decentralized Web Would Give Power Back to the People Online. Tech Crunch. Available at: https://techcrunch.com/2016/10/09/adecentralized-web-would-give-power-back-to-the-people-online/

Hubbard, B. (2020). Federally Chartered Banks and Thrifts May Provide Custody Services for Crypto Assets. The Office of the Comptroller of the Currency (OCC). Available at: https://www.occ.gov/news-issuances/news-releases/2020/ nr-occ-2020-98.html

IBM (2020). Blockchain's Hyperledger Fabric: The flexible blockchain framework that's changing the business world. Available at: https://www.ibm.com/ blockchain/hyperledger (Accessed July 24, 2020).

Janowicz, K., Regalia, B., Hitzler, P., Mai, G., Delbecque, S., Fröhlich, M., et al. (2018). On the Prospects of Blockchain and Distributed Ledger Technologies for Open Science and Academic Publishing. Semantic Web 9, 545-555. doi:10. $3233 / \mathrm{sw}-180322$

Javed, S. (2019). Blockchain: A Viable Solution to Fight Counterfeits? Shutterstock.com. Available at: https://www.worldipreview.com/contributedarticle/blockchain-a-viable-solution

Jones, H. (2020). EU to Introduce Crypto-Assets Regime by 2024, EU Documents Say. The European Union will introduce new rules within four years to make cross-border payments quicker and cheaper through the use of blockchain and crypto assets like stablecoins, two EU documents showed. Reuters. Available at: https://www.reuters.com/ article/us-eu-cryptoassets-idUSKBN2692CP

Kapilkov, M. (2020). A New Cardano-Based Project Is Handling Oracles a Lot Differently Than Chainlink. New York, NY: Cointelegraph. Available at: https:// cointelegraph.com/news/a-new-cardano-based-project-is-handling-oracles-a-lotdifferently-than-chainlink

Kenton, W. (2020). Satoshi Nakamoto. Investopedia. Available at: https://www. investopedia.com/terms/s/satoshi-nakamoto.asp

Kerr, N. L. (1998). HARKing: Hypothesizing after the Results Are Known. Pers Soc Psychol Rev 2, 196-217. doi:10.1207/s15327957pspr0203_4

Kondo, Y., Miyata, A., Ikeuchi, U., Nakahara, S., Nakashima, K. i., Ōnishi, H., et al. (2019). Interlinking Open Science and Community-Based Participatory Research for Socio-Environmental Issues. Current Opin. Environ. Sustain. 39, 54-61. doi:10.1016/j.cosust.2019.07.001

Kurve, A., and Kesidis, G. (2011). Sybil Detection via Distributed Sparse Cut Monitoring," in Proceedings of the 2011 IEEE International Conference on Communications (ICC), Kyoto, Japan. June 5-9, 2011. doi:10.1109/icc.2011. 5963402
Laurent, P., Chollet, T., Burke, M., and Seers, T. (2018). The Tokenization of Assets Is Disrupting the Financial Industry. Are You Ready? Inside Magazine. Available at: https://www2.deloitte.com/content/dam/Deloitte/lu/Documents/ financial-services/lu-tokenization-of-assets-disrupting-financial-industry.pdf

Leible, S., Schlager, S., Schubotz, M., and Gipp, B. (2019). A Review on Blockchain Technology and Blockchain Projects Fostering Open Science. F. Block. 2, 16. doi:10.3389/fbloc.2019.00016

Leshner, R. (2020). Compound Extends DeFi Ethos to Itself, Launches Governance Token. Available at: https://www.coindesk.com/compound-extends-defiethos-to-itself-launches-governance-token (Retrieved September 17, 2020)

Liu, X., Sun, X., and Huang, G. (2019). An Emerging Decentralized Services Computing Paradigm for Big Data Governance: A Position Paper. IEEE Trans. Serv. Comp. 13, 343-355. doi:10.1109/tsc.2019.2951558

Mackey, T. K., Shah, N., Miyachi, K., Short, J., and Clauson, K. (2019). A Framework Proposal for Blockchain-Based Scientific Publishing Using Shared Governance. F. Block. 2, 19. doi:10.3389/fbloc.2019.00019

Malone, T., and Bernstein, M. (2015). Handbook of Collective Intelligence. Cambridge, MA: MIT Press

Mapperson, J. (2020). Cross Chain Is Here: NEO, ONT, Cosmos and NEAR Launch Interoperability Protocols. New York, NY: Cointelegraph. Available at: https://cointelegraph.com/news/cross-chain-is-here-neo-ont-cosmos-and-nearlaunch-interoperability-protocols

Martin, J. (2020). Self-Service Platform Issues Security Tokens Backed by RealWorld Assets. New York, NY: Cointelegraph. Available at: https:// cointelegraph.com/news/self-service-platform-issues-security-tokens-backed-byreal-world-assets

Martin, M. (2018). Reinvent Scientific Publishing with Blockchain Technology. STAT. Available at: https://www.statnews.com/2018/12/21/reinvent-scientificpublishing-blockchain/

McKenna, M. (2020). How ProMED Crowdsourced the Arrival of Covid-19 and SARS. Wired 2020, the Low-Tech Site Run by Health Experts Collects Reports of New Diseases in Real Time. They've Got a Shoestring Budget-And a Stunning Track Record. Available at: https://www.wired.com/story/howpromed-crowdsourced-the-arrival-of-covid-19-and-sars/

Mearian, L. (2020). Sharding: What it Is and Why Many Blockchain Protocols Rely on it. Computerworld 2020, Available at: https://www.computerworld.com/ article/3336187/sharding-what-it-is-and-why-so-many-blockchain-protocolsrely-on-it.html

Miyakawa, T. (2020). No Raw Data, No Science: Another Possible Source of the Reproducibility Crisis. Mol Brain 13, 24. doi:10.1186/s13041-020-0552-2

Nakamoto, S. (2009). Bitcoin: A Peer-To-Peer Electronic Cash System. Cryptography Mailing List. Available at: https://bitcoin.org/bitcoin.pdf.

Ølnes, S., Ubacht, J., and Janssen, M. (2017). Blockchain in Government: Benefits and Implications of Distributed Ledger Technology for Information Sharing. Government Information Quarterly 34, 355-364. doi:10.1016/j.giq.2017.09.007

O’Neal, S. (2020). ETH Miners Will Have Little Choice once Ethereum 2.0 Launches with PoS. New York, NY: Cointelegraph. Available at: https://cointelegraph.com/ news/eth-miners-will-have-little-choice-once-ethereum-20-launches-with-pos

Open Science Collaboration (2015). Psychology. Estimating the Reproducibility of Psychological Science. Science 349, aac4716. doi:10.1126/science.aac4716

Page, M. J., McKenzie, J. E., Kirkham, J., Dwan, K., Kramer, S., Green, S., et al. (2014). Bias Due to Selective Inclusion and Reporting of Outcomes and Analyses in Systematic Reviews of Randomised Trials of Healthcare Interventions. Cochrane Database Syst. Rev. 53 (21), 1362-1370. doi:10. 1002/14651858.MR000035.pub2

Partz, H. (2020). New Bill in Germany Proposes to Digitize Securities with Blockchain. New York, NY: Cointelegraph. Available at: https:// cointelegraph.com/news/new-bill-in-germany-proposes-to-digitize-securitieswith-blockchain

Peng, T. (2020b). A Community-Governed DeFi Platform Makes Crowdfunding Decentralized (Mantra DAO). New York, NY: Cointelegraph. Available at: https://cointelegraph.com/news/a-community-governed-defi-platform-makescrowdfunding-decentralized

Peng, T. (2020a). Blockchain-based IP Protection Application Gets Investment. New York, NY: Cointelegraph. Available at: https://cointelegraph.com/news/ blockchain-based-ip-protection-application-gets-investment

Post, K. (2020). US Banking Regulator Authorizes Federal Banks to Hold Reserves for Stablecoins. New York, NY: Cointelegraph. Available at: https:// 
cointelegraph.com/news/us-banking-regulator-authorizes-federal-banks-tohold-reserves-for-stablecoins

Posth, S. (2020). Decentralization and Scarcity-Blockchain and the Cultural Industries. Medium.com. Available at: https://medium.com/@posth/ decentralization-and-scarcity-blockchain-and-the-cultural-industriesf27068e 82863

Posth, S., and Wittek, K. (2021). Licium/Occp: 0.3-Open Content Certification Protocol. Zenodo. Available at: https://zenodo.org/record/4066529

Posth, S., and Wittek, K. (2020). Whitepaper for the Open Content Certification Protocol (OCCP). Github. Available at: https://github.com/licium/occp/blob/ master/occp-whitepaper.pdf

Rafati Niya, S., Pelloni, L., Wullschleger, S., Schaufelbühl, A., Bocek, T., Rajendran, L., et al. (2019). "A Blockchain-Based Scientific Publishing Platform," in Proceedings of the IEEE International Conference on Blockchain and Cryptocurrency ICBC19, Seoul, South Korea, May 14-17, 2019. doi:10.1109/ BLOC.2019.8751379

Rana, O. S. S. O. G. M. E. (2020). Blockchain Based Framework for Educational Certificates Verification. J. Crit. Rev. 7, 7. doi:10.31838/jcr.07.03.13

Resnik, D. B., and Shamoo, A. E. (2017). Reproducibility and Research Integrity. Account Res 24, 116-123. doi:10.1080/08989621.2016.1257387

Reyes, D. R., Heeren, H. v., Guha, S., Herbertson, L. H., Tzannis, A. P., Ducrée, J., et al. (2021). Accelerating Innovation and Commercialization through Standardization of Microfluidic-Based Medical Devices. Lab on a Chip 21 (1), 9-21. doi:10.1039/D0LC00963F

Roberts, R. (2019). Academic Credentialing Using Blockchain. Medium.com. Available at: https://medium.com/awecademy/academic-credentialing-onthe-blockchain-38da8ee439b2

Rossum, J. V. (2017). Blockchain for Research - Perspectives on a New Paradigm for Scholarly Communication. Digital Science Report. Available at: https://www.digitalscience.com/resources/digital-research-reports/blockchain-for-research/

Rouviere, S. d. 1. (2017). A Practical Example of Curation Markets: An \#ethtrader Token for Curating Good Market Analysis. consensys.net 2021. Available at: https://media.consensys.net/a-practical-example-of-curation-markets-an-ethtradertoken-for-curating-good-market-analysis-6f6f340c6916

Rubin, M. (2017). When Does HARKing Hurt? Identifying when Different Types of Undisclosed Post Hoc Hypothesizing Harm Scientific Progress. Review of General Psychology 21, 308-320. doi:10.1037/gpr0000128

Sharma, R. (2019). Public vs. Private Permissioned Ledgers and Blockchain Standards. forbes.com. Available at: https://www.forbes.com/sites/ forbestechcouncil/2019/06/11/public-vs-private-permissioned-ledgers-andblockchain-standards

Shevchenko, A. (2020). Vitalik Buterin Outlines Next Steps for Ethereum after Beacon Chain Launch. New York, NY: Cointelegraph. Available at: https:// cointelegraph.com/news/vitalik-buterin-outlines-next-steps-for-ethereum-afterbeacon-chain-launch

Siegel, D. (2016). Understanding the DAO Attack. Coindesk 2020. Available at: https://www.coindesk.com/understanding-dao-hack-journalists

Singh, A., Click, K., Parizi, R. M., Zhang, Q., Dehghantanha, A., and Choo, K.-K. R. (2020). Sidechain Technologies in Blockchain Networks: An Examination and State-Of-The-Art Review. J. Network and Comp. Appl. 149, 102471. doi:10. 1016/j.jnca.2019.102471

Singh, N. (2019). Introduction to Permissioned Blockchains. 101blockchains.com. Available at: https://101blockchains.com/permissioned-blockchain/

Smith, G. D., and Ebrahim, S. (2002). Data Dredging, Bias, or Confounding. BMJ 325, 1437-1438. doi:10.1136/bmj.325.7378.1437

Sporny, M., Longley, D., and Chadwick, D. (2019). Verifiable Credentials Data Model 1.0 - Expressing Verifiable Information on the Web (W3C Recommendation). W3C. Available at: https://www.w3.org/TR/vc-data-model/

Surowiecki, J. (2004). The Wisdom of Crowds: Why the Many Are Smarter than the Few and How Collective Wisdom Shapes Business, Economies, Societies, and Nations. New York, NY: Random House.
Swan, M. (2015). Blockchain: Blueprint for a New Economy, Sebastopol, CA: O'Reilly Media Inc.

Titusz, P., and Niederbühl, A. (2020). Iscc/Iscc-Specs: ISCC Reference Code v1.0.5-ISCC: International Standard Content Code. Zenodo. doi:10.5281/ zenodo.4059284

van der Waal, M. B., Dos, S. R. C., Ma, M., Haringhuizen, G. B., Claassen, E., and van de Burgwal, L. H. M. (2020). Blockchain-facilitated Sharing to Advance Outbreak R\&D. Science 368, 719-721. doi:10.1126/science.aba1355

van Rossum, J., and Lawlor, B. (2018). The Blockchain and its Potential for Science and Academic Publishing. Information Services and Use 38, 95-98. doi:10.3233/ isu- 180003

Vigna, P. (2016). Chiefless Company Rakes in More Than $\$ 100$ Million - Group Called DAO Is Running Itself via Computer Code. Wall Street $J$ Available at: https://www.wsj.com/articles/chiefless-company-rakes-in-more-than-100-million1463399393

Voelkl, B., Altman, N. S., Forsman, A., Forstmeier, W., Gurevitch, J., Jaric, I., et al. (2020). Reproducibility of Animal Research in Light of Biological Variation. Nat Rev Neurosci 21, 384-393. doi:10.1038/s41583-020-0313-3

Weiner, J., and McDonald, J. A. (2013). "Three Models of Community-Based Participatory Research," in Issue Brief-Leonard Davis Institute of Health Economics, 18, 2-8. Available at: https://di.upenn.edu/sites/default/files/pdf/ IssueBrief18_5.pdf

Winterbauer, N. L., Bekemeier, B., VanRaemdonck, L., and Hoover, A. G. (2016). Applying Community-Based Participatory Research Partnership Principles to Public Health Practice-Based Research Networks. Sage Open 6, 4. doi:10.1177/ 2158244016679211

Wolfson, R. (2020). Book Review on Don Tapscott's Collaborative "Supply Chain Revolution”. New York, NY: Cointelegraph. Available at: https://cointelegraph.com/ news/book-review-of-don-tapscott-s-collaborative-supply-chain-revolution

Wu, K. (2019). ZK Rollup \& Optimistic Rollup (En). Medium.Com. Available at: https://medium.com/coinmonks/zk-rollup-optimistic-rollup-70c01295231b

Yang, C. (2020). Defining the New AI-Powered SaaS: Science as a Service. Medium.com. Available at: https://medium.com/swlh/ml4sci-8-defining-thenew-ai-powered-saas-science-as-a-service-d5e66a4aa8bf

Zero-Knowledge (2020). Non-interactive Zero-Knowledge Proof. Available at: https://en.wikipedia.org/wiki/Non-interactive_zero-knowledge_proof (Retrieved September 7, 2020)

Zhang, F., Cecchetti, E., Croman, K., Juels, A., and Shi, E. (2016). “Town Crier: An Authenticated Data Feed for Smart Contracts," in CCS'16: Proceedings of the 2016 ACM SIGSAC Conference on Computer and Communications, Vienna, Austria, October 24-28, 2016. 270-282. doi:10.1145/2976749.2978326

Zuiderwijk, A., and Spiers, H. (2019). Sharing and Re-using Open Data: A Case Study of Motivations in Astrophysics. Inter. J. Inform. Manag. 49, 228-241. doi:10.1016/j.ijinfomgt.2019.05.024

Conflicts of Interest: ME was employed at Akasha Foundation. SB is the sole proprietor and owner of Blockchain for Science GmbH. SP is the sole proprietor and owner of Posth Werk BV.

The remaining authors declare that the research was conducted in the absence of any commercial or financial relationships that could be construed as a potential conflict of interest.

Copyright (® 2021 Ducrée, Etzrodt, Bartling, Walshe, Harrington, Wittek, Posth, Wittek, Ionita, Prinz, Kogias, Paixão, Peterfi and Lawton. This is an open-access article distributed under the terms of the Creative Commons Attribution License (CC $B Y)$. The use, distribution or reproduction in other forums is permitted, provided the original author(s) and the copyright owner(s) are credited and that the original publication in this journal is cited, in accordance with accepted academic practice. No use, distribution or reproduction is permitted which does not comply with these terms. 